\title{
Simulation of Sweep-Jet Flow Control, Single Jet and Full Vertical Tail
}

\author{
Robert E. Childs, ${ }^{*}$ Paul M. Stremel, ${ }^{\dagger}$ Joseph A. Garcia, ${ }^{\ddagger}$ \\ James T. Heineck, ${ }^{\S}$ Laura K. Kushner, ${ }^{\llbracket}$ Bruce L. Storms"
}

I. Abstract

This work is a simulation technology demonstrator, of sweep jet flow control used to suppress boundary layer separation and increase the maximum achievable load coefficients. A sweep jet is a discrete Coanda jet that oscillates in the plane parallel to an aerodynamic surface. It injects mass and momentum in the approximate streamwise direction. It also generates turbulent eddies at the oscillation frequency, which are typically large relative to the scales of boundary layer turbulence, and which augment mixing across the boundary layer to attack flow separation. Simulations of a fluidic oscillator, the sweep jet emerging from a nozzle downstream of the oscillator, and an array of sweep jets which suppresses boundary layer separation are performed. Simulation results are compared to data from a dedicated validation experiment of a single oscillator and its sweep jet, and from a wind tunnel test of a full-scale Boeing 757 vertical tail augmented with an array of sweep jets. A critical step in the work is the development of realistic time-dependent sweepjet inflow boundary conditions, derived from the results of the single-oscillator simulations, which create the sweep jets in the full-tail simulations. Simulations were performed using the computational fluid dynamics (CFD) solver Overflow, with high-order spatial discretization and a range of turbulence modeling. Good results were obtained for all flows simulated, when suitable turbulence modeling was used.

\section{Introduction}

NASAs Environmentally Responsible Aviation (ERA) Project ${ }^{1}$ explores concepts and technologies that have the potential to reduce aviation's adverse environmental effects. The Project covers topics in materials, airframe concepts, propulsion, and flow control. In the area of flow control, there are topics on natural laminar flow and boundary layer separation control. One such technology is the Active Flow Control (AFC) sweep jet, which can be used to delay boundary layer separation, increase the maximum aerodynamic loading, and enable the design of smaller and lighter structures with lower drag and fuel burn. Effective flow control may also be used to develop new air vehicle concepts, simplify existing ones and improve vehicle performance. Many experimental studies have evaluated the use of sweep jets for flow control, ${ }^{3,5,14,21,23}$ and they have demonstrated the effectiveness of sweep jets at delaying stall on a lifting surface to higher angles of incidence, yielding higher maximum lift coefficients.

The sweep-jet AFC concept combines elements of a Coanda jet and of periodic forcing, which are both, independently, effective means of flow control under some conditions. When the mean sweep-jet flow direction differs from the local freestream, the jet also creates mean streamwise vorticity, like a powered vortex generator. The effectiveness of sweep-jet flow control may result from multiple physical mechanisms. The oscillating jet generates rapid time-averaged spreading of the jet and elevated turbulent shear stress, in the

\footnotetext{
*Senior Research Scientist, Science and Tech. Corp. @NASA Ames, robert.e.childs@nasa.gov

†Senior Research Scientist, Science and Tech. Corp., @NASA Ames, AIAA Member

${ }^{\ddagger}$ NASA Ames Research Center, AIAA Senior Member

$\S$ NASA Ames Research Center, AIAA Member

"ACI/NASA Ames Research Center

"NASA Ames Research Center
} 
plane of the oscillations. Less well understood is the nature of turbulence in the wall-normal direction, and how that turbulence interacts with the boundary layer and freestream above the sweep jet. The local freestream is an important source of energy available to energize the near-wall boundary layer, and hence delay separation. Thus, interactions of the sweep jets with the outer flow are expected to be significant to sweep-jet AFC performance.

The vertical tail on the Boeing 757 ecoDemonstrator $^{2}$ is the focus of the work reported here. On a modern twin-engine commercial transport aircraft, the vertical tail is sized to provide yaw loads in engine-out takeoff scenarios, and it is typically larger than needed for normal flight. System studies estimate that fuel consumption may be reduced by as much as a percent by using a smaller tail, made possible by AFC. The ecoDemonstrator tail is augmented with 37 sweep jet nozzles positioned in a uniform array just ahead of the rudder's hingeline on the starboard side, which are intended to keep the rudder flow attached at high rudder deflection angles. (This test vehicle and tail are asymmetrical, using sweep jets to steer left.) The tail's performance was evaluated in a unique wind tunnel experiment conducted in the $40^{\prime} \times 80^{\prime}$ test section of the National Full-Scale Aerodynamics Complex (NFAC) operated by the Arnold Engineering Development Center (AEDC) at NASA's Ames Research Center. The augmented tail demonstrated enhanced side force capability in the wind tunnel test, ${ }^{3,21}$ and flight testing was conducted by Boeing over the Strait of Juan de Fuca, in 2015. The configuration selected for CFD simulations achieved a goal for side-force improvement, with only the lowest 31 jets powered, leaving the upper 6 jet nozzles unpowered.

The demonstrated success of sweep-jet flow control leads to the next challenge: how to develop good designs of products that use this AFC technology. At a time when the aerospace industry relies, increasingly and with good justification, on CFD-based design strategies, the aerodynamics of sweep-jet AFC are so complex that CFD accuracy and utility may be greatly diminished. Accurately simulating these highly unsteady sweep jets is challenging for CFD, and it was even thought to be unachievable by current CFD methods. The problem
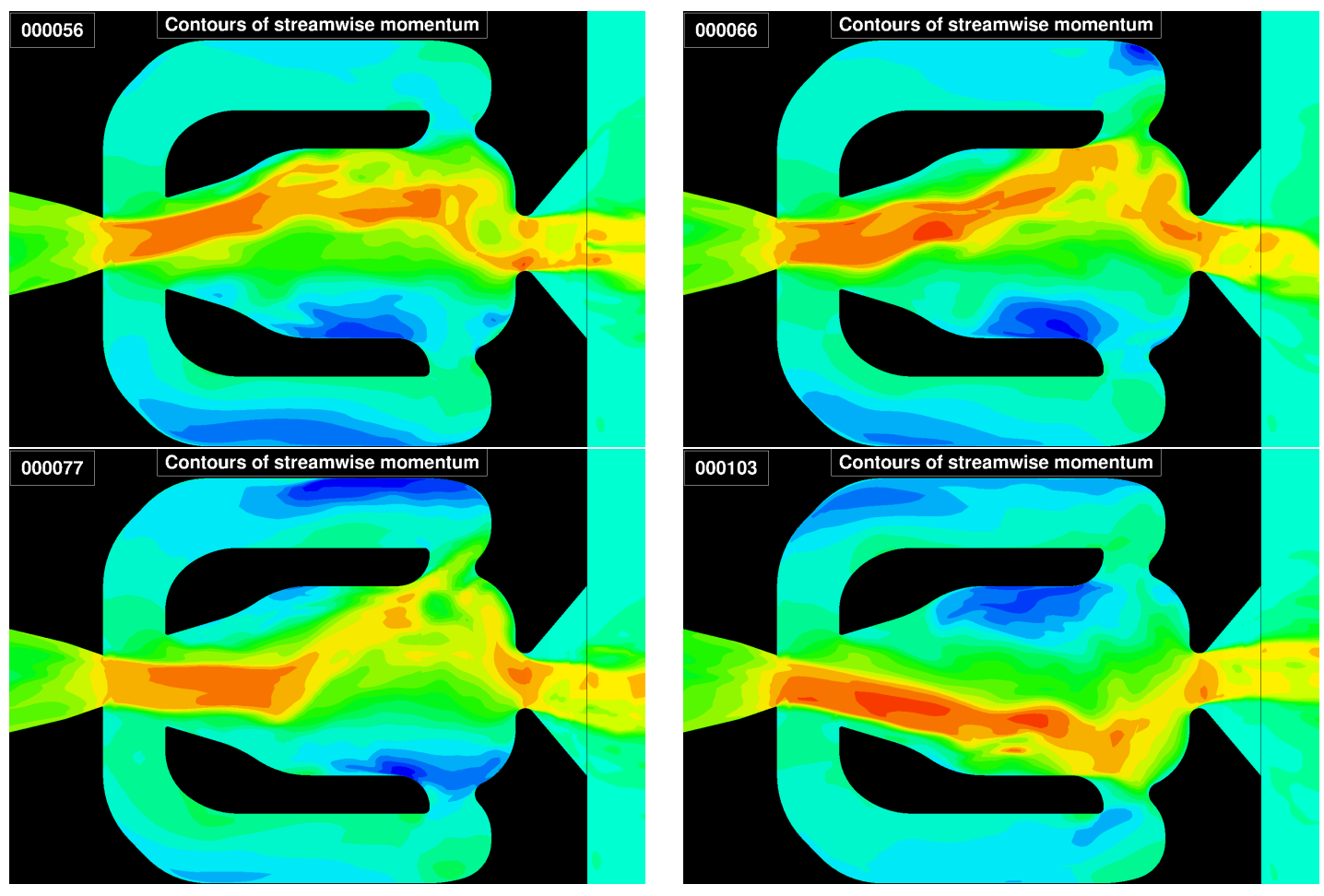

Figure 1: Streamwise momentum in fluidic oscillator and near-field external jet in a time sequence (frame number in upper left corner) covering half of an oscillation period. Supply pressure is steady at $\Delta p / p_{\infty}=$ $2.585, p_{\infty}=1 b a r$. Images are from CFD, with DES turbulence modeling. Primary flow is from left to right (red contours) in central passage, while reversed flow (blue contours) occurs in central passage and side-loops. Observe the mirror-image phase reversal of the central-jet and reversed flow in side-loops between the first and last frames. 
is very computationally intensive due to the highly unsteady AFC sweep jets and its induced turbulence, including the wide range of length and time scales of the turbulence and the mean flow. ${ }^{4,7-9,14,15,18}$ If CFD is ineffective for sweep jet flows, this could force the design optimization work for all features associated with sweep-jet AFC back into the machine shop and wind tunnel, which may delay the adoption of this technology and its environmental benefits. Thus, the ability to simulate AFC aerodynamics is relevant to realizing the environmental goals of the ERA Project. While the main goal of this AFC wind tunnel test was to demonstrate the technology's effectiveness on the full scale tail, the aerodynamic data are used here for validation of simulation methods.

The physics of the fluidic oscillation are briefly reviewed as background for determining about how to best run the simulations. The sweep jets studied here originate from an internal fluidic oscillator, like the one shown in Fig. 1. Four frames during one half of an oscillation period are used to highlight key elements of the fluidic oscillator's design and flow physics. (1) Structurally, the oscillator is an internal flow left of the right-most nozzle; to the right of this nozzle, it has a back-plane but no cover-plate, so is open to the local freestream (and here, toward the reader's viewpoint). Another view of the oscillator is given in Fig. 3. (2) Steady high pressure air is supplied to the left-most passage, which flows through a control nozzle into the central chamber, and then exhausts through the right-most nozzle. (3) In the first frame (labeled 000056), the jet flowing through the central passage has a positive perturbation angle, and it impinges on the opening into the upper side passage, increasing the pressure there. (4) The elevated pressure causes the flow in that side passage to accelerate, and after a time lag, to develop a significant reversed flow velocity, as in the second and third frames $(000066,000077)$. (5) The side-passage flow drives the central jet to the opposite side of the central passage, as in the forth frame (000103), which is essentially a mirror image of the first frame. These stages constitute one half of a full period of the fluidic oscillation. (6) The instantaneous external jet is created by flow through the right-most nozzle. Its velocity is governed by the mean streamwise pressure drop plus the fluctuating pressure gradient from the impact pressure of the oscillating interior jet. The external jet does not diverge significantly; instead, it retains the nozzle's cross-section, approximately, and its flow angle oscillates between the two side-walls. This oscillating external jet is the sweep jet studied in the present work.

While the oscillator flow is compressible at the pressure ratios considered here, the key oscillation physics is dominated by inviscid incompressible convective fluid dynamics. The acoustic propagation time is roughly an order of magnitude faster than convective processes, for the observed behavior and dimensions of the device being used. It is probable that turbulent entrainment of fluid between the central jet and an adjacent wall plays a significant role when the two are in proximity. Entrainment will help the central jet stick to the closest wall, and turn the corner into the side-passage. One can speculate that this will help energize flow into the side passages, and delay the central jet releasing from a wall due to forcing from the side-loop flow. Both effects may influence the frequency and strength of the oscillation.

The work described here is a technology demonstration for simulations of sweep-jet AFC flows. The simulations encompass the entire relevant flow path of sweep jet aerodynamics, from "end-to-end," starting with steady flow into a supply pipe upstream of the oscillator plenum and ending with the $40^{\prime} \times 80^{\prime}$ wind tunnel test section. However, this full range of length and time scale is not done in a single monolithic simulation. It is split into two parts: a single oscillator (and its near-field sweep jet), and the external domain of the full tail, starting from the sweep-jet nozzle throat. The nozzle-throat boundary conditions which power all of the external flow sweep-jets are derived from the single-jet simulations, and they retain most of the complex fluid mechanics of the simulated flow through the oscillator and nozzle. The decision to approximate sweep-jet inflow with boundary conditions (BCs) has important implications for the simulations' computational cost, accuracy and range of applicability, which are discussed below.

The three middle sections of this paper mirror these divisions in the simulation strategy. One section involves CFD and measurements of a single oscillator and its sweep jet, in a dedicated wind tunnel experiment in the Fluid Mechanics Laboratory (FML) at NASA's Ames Research Center. The test involved an oscillator and sweep jet that were flight-scale $\left(0.5^{\prime \prime}\right.$ across vertical, as viewed in Fig. 1 and $0.25^{\prime \prime}$ deep) and operated at supply pressures up to and exceeding the full-tail test. The next section covers the development and testing of the sweep-jet nozzle-throat BCs. These BCs consist of data extracted from the history from singlejet simulations, and boundary-condition software which "plays back" the recorded history, in the correct orientation, at the correct frequency, and onto the external-flow grid. The final section describes the results 
of using those BCs in the full Boeing 757 tail simulations, and compares simulation results to data from the full-tail wind tunnel test.

\section{Simulation Methods}

This section describes the relevant details of the CFD simulation methods. Simulations were performed using Overflow ${ }^{17}$ version $2.2 \mathrm{f}$ on a system of overset structured grids, with grid connectivity generated by Pegasus. ${ }^{16}$ The code was modified only to include an imposed-time-dependent boundary condition for the sweep jets.

Good spatio-temporal discretization accuracy is needed to resolve the relatively small scales and high frequencies of the sweep jets. Spatial discretization of the Euler terms is done with Overflow's fifth-order WENO-M scheme throughout the computational domain, except within a single grid zone at the base of the rudder where third-order was used to suppress numerical instability. Third-order upwind discretization is used for convection of turbulence variables, and second-order central differencing is used for diffusion terms. The same discretization is used in all simulations. Time integration for all simulations is done using the "time-accurate mode" with inner iterations inside each time step. Flows that are intended to reach a steady state are run with first-order time integration, a large outer time step and a small number of inner iterations. This choice of parameters renders the simulations non-time-accurate, but they generally converge rapidly in a quasi-physical manner to a steady state. Flows that involved oscillating sweep jets are run with second-order integration, a suitably small time step, and enough inner iterations to achieve good inner-iteration convergence of the integrated loads, as in Fig. 2. The inner-iterative solver is the SSOR algorithm, with a spatially varying step and settings of $C F L_{\min } \sim 10.0$ and $C F L_{\max } \sim 25.0$. SSOR is also used for the turbulence model equations.

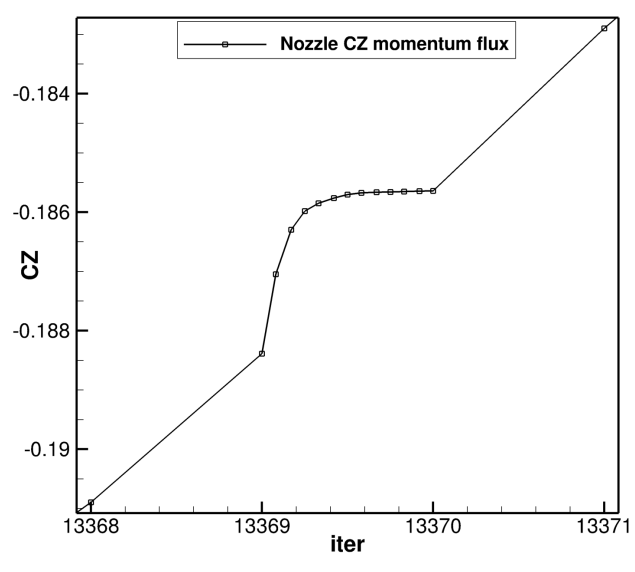

Figure 2: Example of the convergence of the sweep-jet nozzle-throat lateral momentum flux coefficient $(\mathrm{CZ})$ during the inner iterations of a time step (iter). The inner iteration must converge to its steady state to achieve intended outer-time-step accuracy. This level of inner-convergence is typical.

The grid spacing and time step have been refined, iteratively but not systematically, in response to perceived needs in prior simulations with inferior resolution. Early in the work, qualitative grid refinement and timestep convergence studies were done with a single-jet simulation that involved both the interior oscillator and external jet. The grid refinement goal was to achieve 15 or more grid points across shear layers in critical regions of the flow (e.g., the central flow-path in Fig. 1) in RANS simulations. Because of shear layer dynamics, a relatively fine grid is needed across the oscillator's entire central flow-path. The same grids are used for RANS and D/DES simulations. A time step of $5 \mu s$ was selected for the oscillator and single sweep jet flows, based on a time-step study using the final grid system. The number of inner iterations was selected based on the inner-convergence of relevant integrated loads. The time-integration achieves its full outer time step accuracy only when the inner iterations converge to their asymptotic value. An example of sub-iteration convergence is shown in Fig. 2. The lateral momentum flux coefficient for the nozzle throat, which is the most important result of the single-jet simulations, is very close to its asymptotic value after twelve subiterations. Overflow's loads-integration was set to report the inner convergence infrequently (e.g. every $40^{\text {th }}$ time step), and this coarse running record of the inner convergence was reviewed occasionally throughout the work.

The use of zonal turbulence modeling is anticipated, as the flows studied here have distinctly different aerodynamics in different regions. Overflow has a range of turbulence modeling options, but this work considered only the SST-RANS ${ }^{11,12}$ and SST-D/DES models. Zonal turbulence modeling is easily accomplished in Overflow (within a family of models), by setting the "DES switch" appropriately in each grid zone. One part of the zonal strategy is to explicitly select the SST-RANS model where the flow is expected to involve steady attached boundary layers. Where the flow may be separated or unsteady due to sweep jets, all of the RANS 
and D/DES options were treated as viable, to be judged in comparison to experimental measurements. The relative merits of these turbulence modeling options are discussed with the simulation results.

Conventional boundary conditions are used for all solid walls and the inflow/outflow planes in the wind-tunnel simulations. The sweep-jet inflow boundary conditions are discussed in their own section, below.

All sweep jets simulated in this work have the same physical dimensions, operate at comparable pressure ratios and Mach numbers, and have similar time scales. Thus, lessons learned in the single-jet work are relevant to the full-tail simulation. However, the full-tail simulations involve the far-downstream development of the sweep jets, where the dominant length scales are larger than in the oscillator and in the jet's near field.

An important part of these simulations is nature of the data recorded for aerodynamic analysis. Integrated loads are always saved. For simulation running in what may later be recognized as the asymptotic state, specific points, lines, planes or volumes of data were recorded. These could be saved as instantaneous, or mean and fluctuation data. Simulations were run over many PBS job submissions. For each PBS job, Overflow was directed to save the specified results, over just that PBS job. When the loads were adequately converged, over a sufficient time, the appropriate shorter-time averages were then combined to give a single set of mean and fluctuation results, representative of the asymptotic unsteady solution.

\section{Single Oscillator and Sweep Jet}

A critical part of the work has been the collaboration between the simulation and experimental studies of a single sweep jet. This work was essential for establishing and validating CFD methods, and for developing the sweep-jet boundary conditions for the external-only flows. Experimental studies of the single sweep-jet were performed in the Fluid Mechanics Lab (FML) at the NASA Ames Research Center. ${ }^{10}$ The single sweep-jet apparatus is shown in Fig. 3. The sweep jet nozzle is located far enough above the tunnel's bottom wall that the influence of the tunnel-wall on the near-field external jet is believed to be small. The primary experimental measurements used to characterize the flow and to assess the simulations' accuracy are the unsteady pressure at the nozzle throat, and PIV (particle imaging velocimetry) of the exterior sweep jet. Schlieren images enable qualitative evaluation of the instantaneous external jet structure.
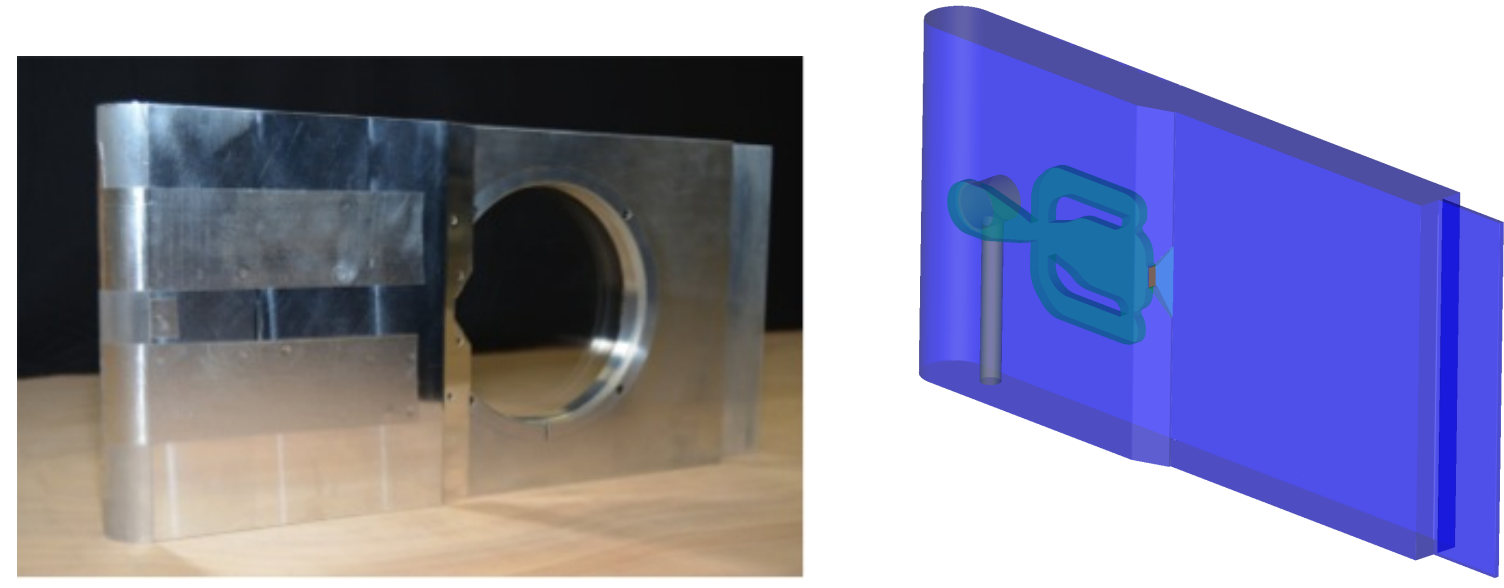

Figure 3: Photograph of single sweep jet test article (left), and see-through image of CFD surface definition (right). Jet air supplied via a pipe through the tunnel floor. Sweep jet nozzle is in the "notch" at the left edge of the window that provides PIV and Schlieren access. External flow is from left to right. A trailing edge splitter plate stabilizes the downstream wake flow.

The flow conditions for this test typically involved a freestream Mach number of $M_{\infty}=0.15$, with jet plenum pressures up to $48 p$ sig. Many of the measurements were taken at a plenum pressure of $\Delta p / p_{\infty}=2.585$, $p_{\infty}=1$ bar and $M_{\infty}=0.15$. At this supply pressure and with modest internal losses, the external sweep jet is supersonic. 


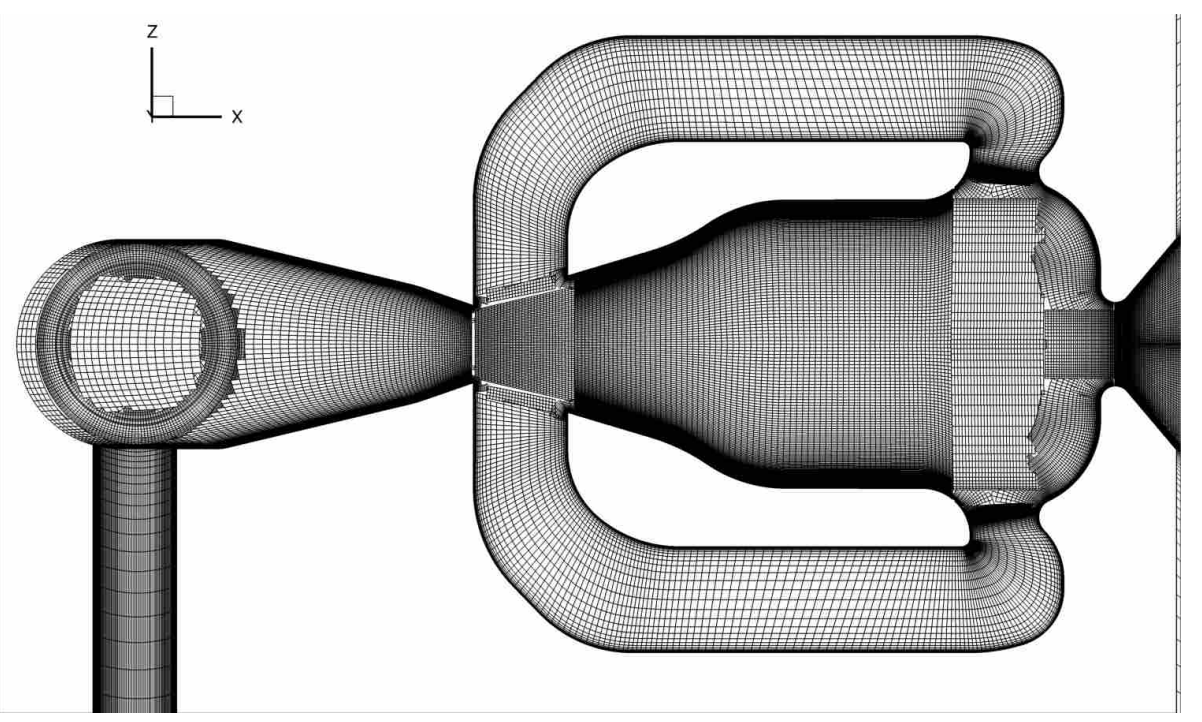

Figure 4: Grid used in oscillator. The grid used for the results presented here had 36M grid points, with about half of those used for the oscillator interior flow.

The oscillator interior grid, shown in Fig. 4, was repeatedly refined until the solution appeared to be adequately resolved, with $\sim 15$ grid points across the central jet's shear layers in RANS simulations. Because of its special grid-convergence properties, DES simulations were not judged in this manner. The grid is also well refined near the walls of the central passage, and in the region of the exterior nozzle.

Figure 5 gives three images of the instantaneous sweep-jet field, selected "by eye" to be approximately at the same phase. The jet supply pressure is $\Delta p / p_{\infty}=2.585, p_{\infty}=1 b a r$ for all three images. The PIV and CFD velocity data have freestream Mach number $M_{\infty}=0.15$ The RBOS image (retroreflective backgroundoriented Schlieren) from Kushner ${ }^{10}$ has $M_{\infty}=0$ to mitigate blurring from tunnel vibration. Key features to note in these images include: (1) the similarity of the shape of the jet's arc, (2) the tendency to have a compact near-field jet and a spreading downstream jet, (3) CFD and RBOS show about 5 shock cells, while shock cells are not visible in the PIV data, (4) PIV exhibits higher velocity outside of the jet, relative to CFD. The comparison of instantaneous flow images can only suggest qualitative similarity, as there are significant cycle-to-cycle differences in the flow. Kushner ${ }^{10}$ also compared CFD and experimental mean flow images, but these are also qualitative.

High-frequency pressure data from near the nozzle exit were used to assess the accuracy of the oscillator interior simulations and to select the turbulence modeling for the interior flow. A Kulite pressure port located adjacent to the upper side-wall of the nozzle throat $\left(0.045^{\prime \prime}\right.$ downstream and $0.219^{\prime \prime}$ offset from the center of the throat) was selected for the highest-frequency Kulite measurements, because it reflects interior processes and the initial exterior deflection of the sweep jet, which is critical to the exterior flow. The CFD image in Fig. 5 includes pressure contour lines, and the pressure at the Kulite port is elevated when the jet is deflected toward the upper wall. The Kulite signal was obtained at $100 \mathrm{kHz}$ and then passed through a $25 \mathrm{kHz}$ low-pass filter. Simulations of the interior were run using the SST-RANS and the SST-DES models. The CFD time step was $200 \mathrm{kHz}^{-1}$ and the sampling rate was $100 \mathrm{kHz} z^{-1}$. It is estimated that signals below $\sim 10 \mathrm{kHz}$ are very well time-resolved, based on the properties of the time integration.

Figure 6 compares the measured and simulated pressure histories. The CFD time was adjusted to align the phase with the experiment, as absolute time is irrelevant. The sample period is representative: these images give neither the worst nor best comparisons that might be found throughout the full time records. The left image gives the pressure history for the RANS simulation over $\sim 8$ periods, while the right image gives DES results over $\sim 3$ periods. Four characteristics of the pressure signals are noted. (1) There is significant cycle-to-cycle variability in the period and amplitude of the pressure signal, in both CFD and experiment. (2) There is good net phase agreement over the full interval is this figure, as the pressure rise at $t=0.101$ and $t=0.131$ shows good coincidence between CFD and experiment. Spectra given below also demonstrate good accuracy for the dominant frequency. (3) The CFD pressure fluctuation amplitude 

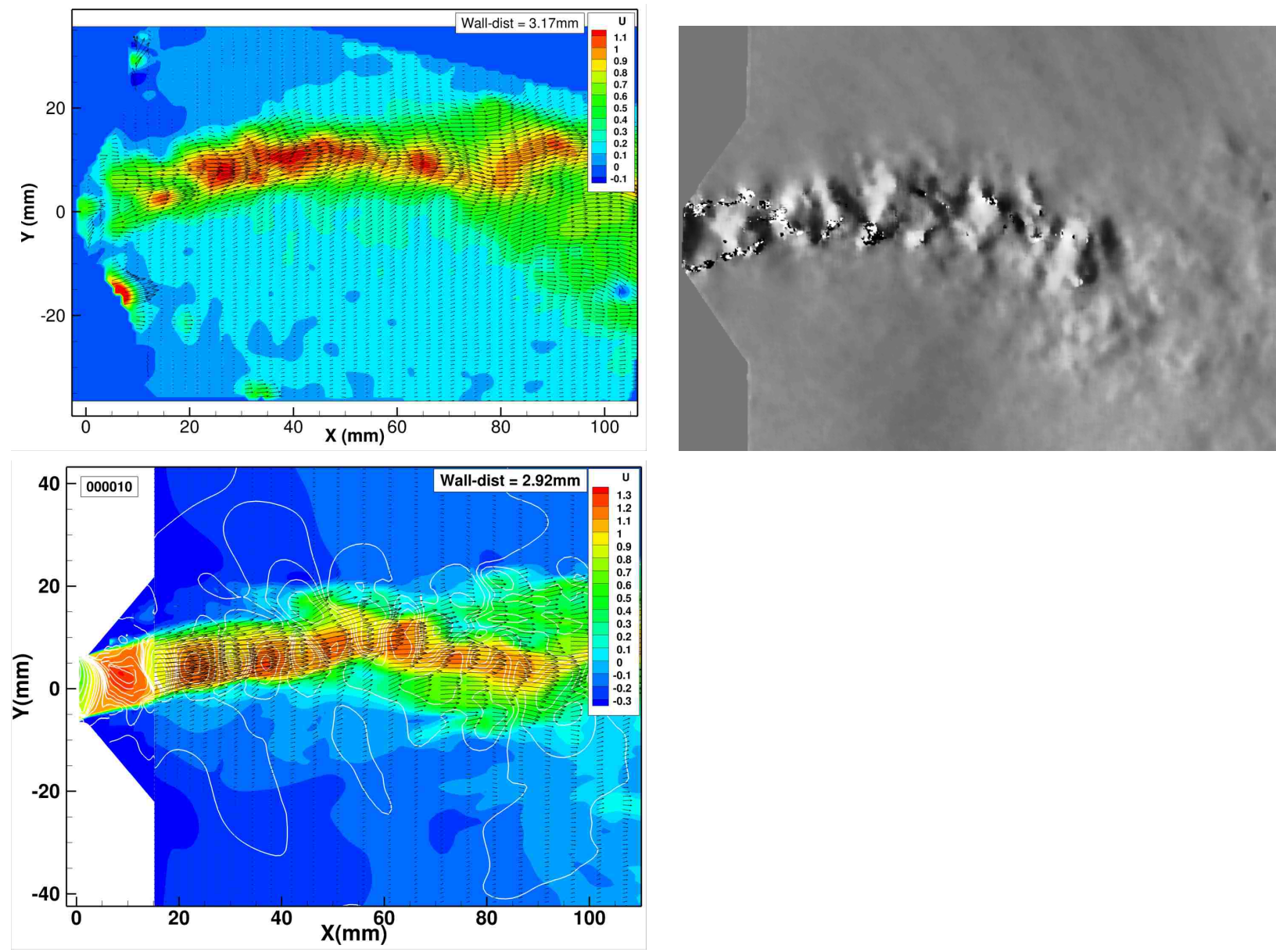

Figure 5: Visualization of instantaneous sweep jet at a supply pressure $\Delta p / p_{\infty}=2.585, p_{\infty}=1 b a r$. Experimental PIV and Schlieren images, top row; Simulation with RANS modeling for interior flow and DDES for exterior flow, bottom image. Flood color contours of streamwise velocity U, normalized by sound speed $c=335 \mathrm{~m} / \mathrm{s}$; velocity vectors; white line contours of pressure in CFD results. Jet phase was selected for approximate similarity in all images.

is noticeably lower than the experiment. The strength of the pressure signal exhibits strong dependence on turbulence modeling, with the RANS simulations being superior to DES for this pressure comparison. (4) The shape of the RANS and experimental pressure signals have many similarities: both dwell longer at low pressure and spend less time at high pressure, they have relatively rapid transitions between low and high pressure, and the qualitative nature of the high-frequency "jaggedness" in the signals is similar.

Several characteristics of the DES results are inferior to the RANS results. While the peak-to-peak pressures are comparable to RANS results, those peaks are reached by pressure fluctuations at frequencies well above the dominant frequency, and there is too little energy in the dominant frequency. Also, the character of the DES waveform differs from experiment and RANS: the large, rapid transition events between low and high pressure are generally absent; in their place is a weaker oscillation at the primary frequency plus significant high-frequency energy. However, the DES short-time peak-to-peak pressure signal better matches the experiment, when the signal is dwelling near $P / P_{\infty}=1$. For simulations of the full tail, we expect that the strength of the signal at the dominant frequency is of greatest importance, and that the RANS oscillator simulation is preferred.

Spectra from experiment and CFD are shown in Fig. 7. This Figure compares spectra of the measured and RANS-simulated pressures, and the nozzle throat lateral momentum flux which is directly related to the jet sweep angle. The experiment has a narrow primary peak at $f_{1} \sim 238 \mathrm{~Hz}$ and harmonics at $f_{2} \sim 475 H z$ and $f_{3} \sim 710 \mathrm{~Hz}$, which are down $\sim 16 d B$ and $\sim 30 d B$ respectively from the primary peak. The pressure 

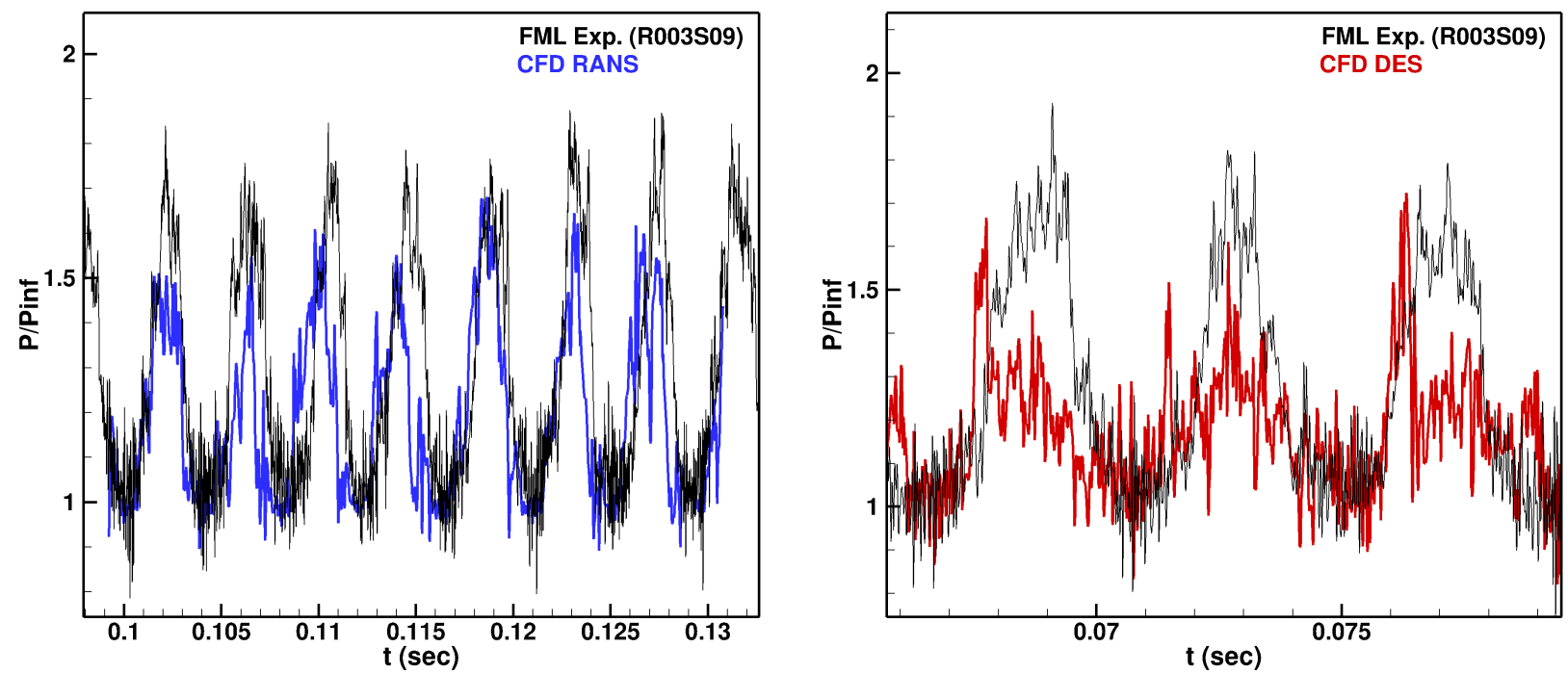

Figure 6: Time history of $P / P_{\infty}$ from FML Kulite measurements at the side-wall of the nozzle throat, from RANS and DES simulations.

spectrum from RANS-CFD interior simulation appears to have two essentially merged peaks at $\sim 220 \mathrm{~Hz}$ and $\sim 238 \mathrm{~Hz}$. At an earlier stage of the work, the dominant frequency appeared to be $\sim 220 \mathrm{~Hz}$, and much of the subsequent analysis was performed using that time scale for the sweep jet. Also included is the spectrum of the nozzle's $z$-momentum flux coefficient, CZ, which has a better defined peak at $\sim 238 \mathrm{~Hz}$. (The CZ amplitude was artificially matched to the experimental PSD to help see the peak in the spectrum.) One of the challenges in spectral analysis of CFD results is the very long run times that are needed to obtain good frequency resolution. The frequency resolution in the experimental data is $\sim 1.5 \mathrm{~Hz}$. The CFD simulation ran for total duration of $\sim 0.2 \mathrm{~s}$, and the best possible resolution of the spectra is $\sim 5 \mathrm{~Hz}$. That resolution is available only in the integrated loads, which were recorded from the start on the simulation. Due to the Hamm windowing $(1-\cos )$, the start-up transient is strongly deemphasized from the spectrum; a side effect of windowing is some degradation of the actual frequency resolution. The CFD pressure data needed for comparison with the Kulite data were recorded for roughly the last $75 \%$ of the total simulation, and have a precision of $\sim 7 \mathrm{~Hz}$, which is further degraded by windowing. The higher harmonic peaks seen in the
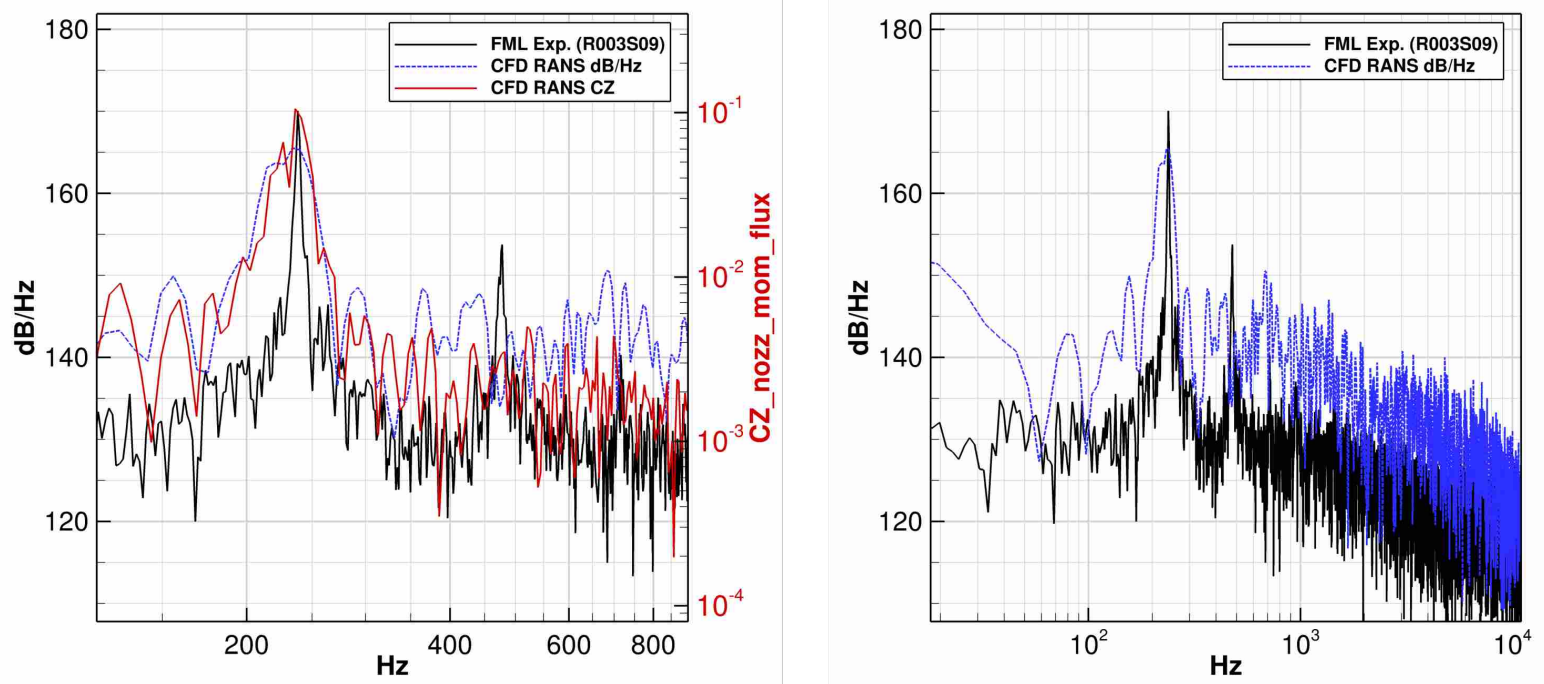

Figure 7: Spectra of nozzle throat pressure, measured in FML test and CFD, and CFD throat momentum flux coefficient (see text). Details near peak in spectrum, left, and full resolved spectrum, right. 


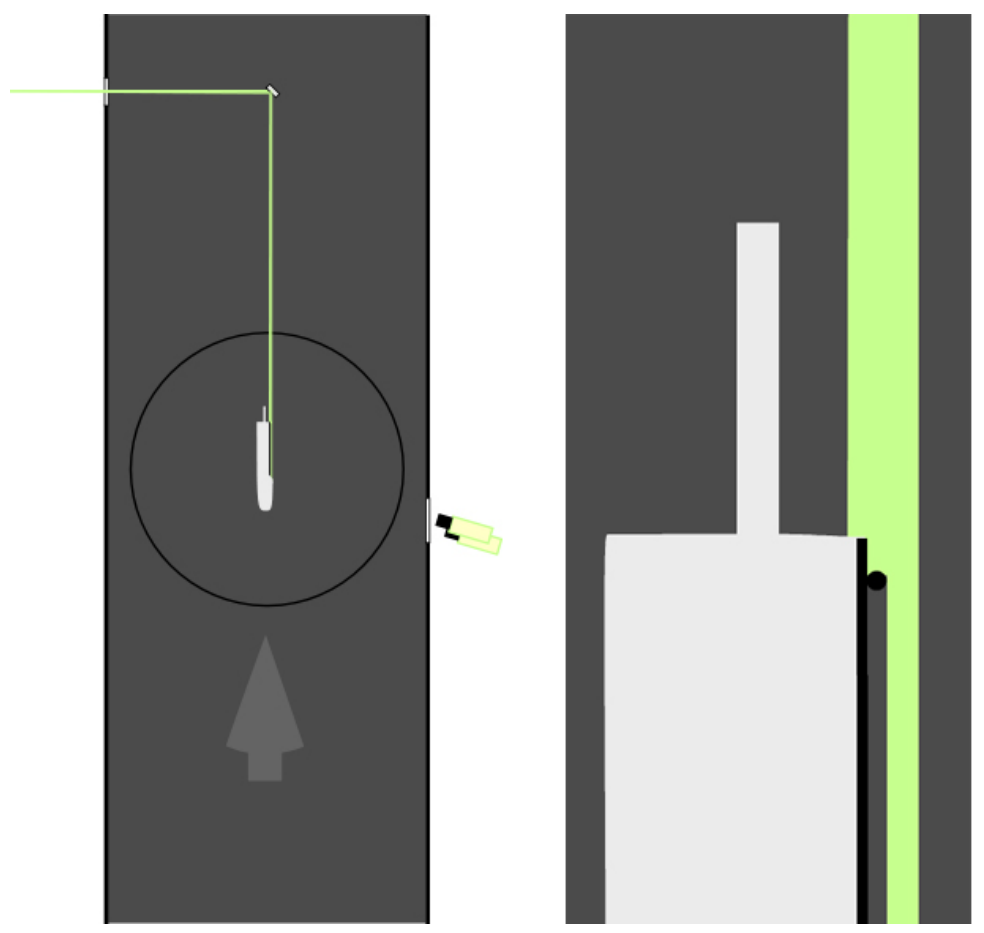

Figure 8: Top view of sweep jet test article and PIV laser sheet, relative to view in Fig. 3, showing laser path into the wind tunnel test section and camera's positions (left), and the bump used to shadow the laser and regulate the laser sheet's thickness (right).

experimental data do not appear in the CFD results.

The accuracy of the exterior flow simulation is judged by comparison to particle imaging velocimetry (PIV) measurements of the mean and averaged fluctuating velocities, within the PIV laser sheet shown in Fig. 8, and above the glass window shown in Fig. 3. Figure 8 shows the orientation of the PIV laser sheet and the sweep jet model. The surface of the model downstream of the jet was covered with black Mylar to create a pure black background. The laser diverged slightly which required a $1 \mathrm{~mm}$ bump to be inserted on the surface of the model in order to shadow the surface of the Mylar from the laser.

The PIV data were acquired using the LaVision DaVis image acquisition software and controllers, and processed with LaVision Flowmaster version 8.2. The laser was a dual-beam Quantronix Darwin Duo Nd:YLF, with each beam operating at $2 k H z$. The delay of one to the other was $10 \mu s$. The cameras were Phantom V641s which operated at $4 k H z$ with a windowed resolution of $764 \times 651$. Seeding was introduced into both the free stream and the sweep-jet feed line using a distribution rake at the inlet of the tunnel and a pressure plenum that was piped into a venturi, respectively. The seed generator was a Laskin-nozzle atomizer, which generated particles with a size distribution of 0.7 microns $+/-.04$ microns. The data were processed using an iterative multi-window scheme which started at 96 pixels square and finished at 24 pixels square. The core of the jet was processed with a pre-shift of 7 pixels to accommodate the large displacements of the flow field. Fig. 5 illustrates that PIV did not acquire consistently useful data very near the sweep jet nozzle, as the instantaneous PIV velocity image does not appear approximately similar to that shown in CFD and Schlieren images. Downstream of about $\sim 20 \mathrm{~mm}$, all three images appear to represent the same flow field, and the PIV statistical data are presented only in the domain where they are believed to be accurate.

Figure 9 gives plots of the mean streamwise and cross flow velocities, $U$ and $V$, and the turbulent shear stress, $\overline{u^{\prime} v^{\prime}}$, which is the vertical transport of the streamwise momentum, in the frame of the figure. Velocities are normalized by the freestream sound speed $c=335 \mathrm{~m} / \mathrm{s}$. The simulation uses RANS turbulence modeling inside the oscillator and DDES modeling for the exterior flow. The PIV measurement plane is nominally $1 \mathrm{~mm}$ wide, centered in the jet throat and thus $3.175 \mathrm{~mm}\left(1 / 8^{\prime \prime}\right)$ from the wall. The CFD data are from the grid plane $2.92 \mathrm{~mm}$ from the wall, which is the closest to the center of the measurement plane, of the 

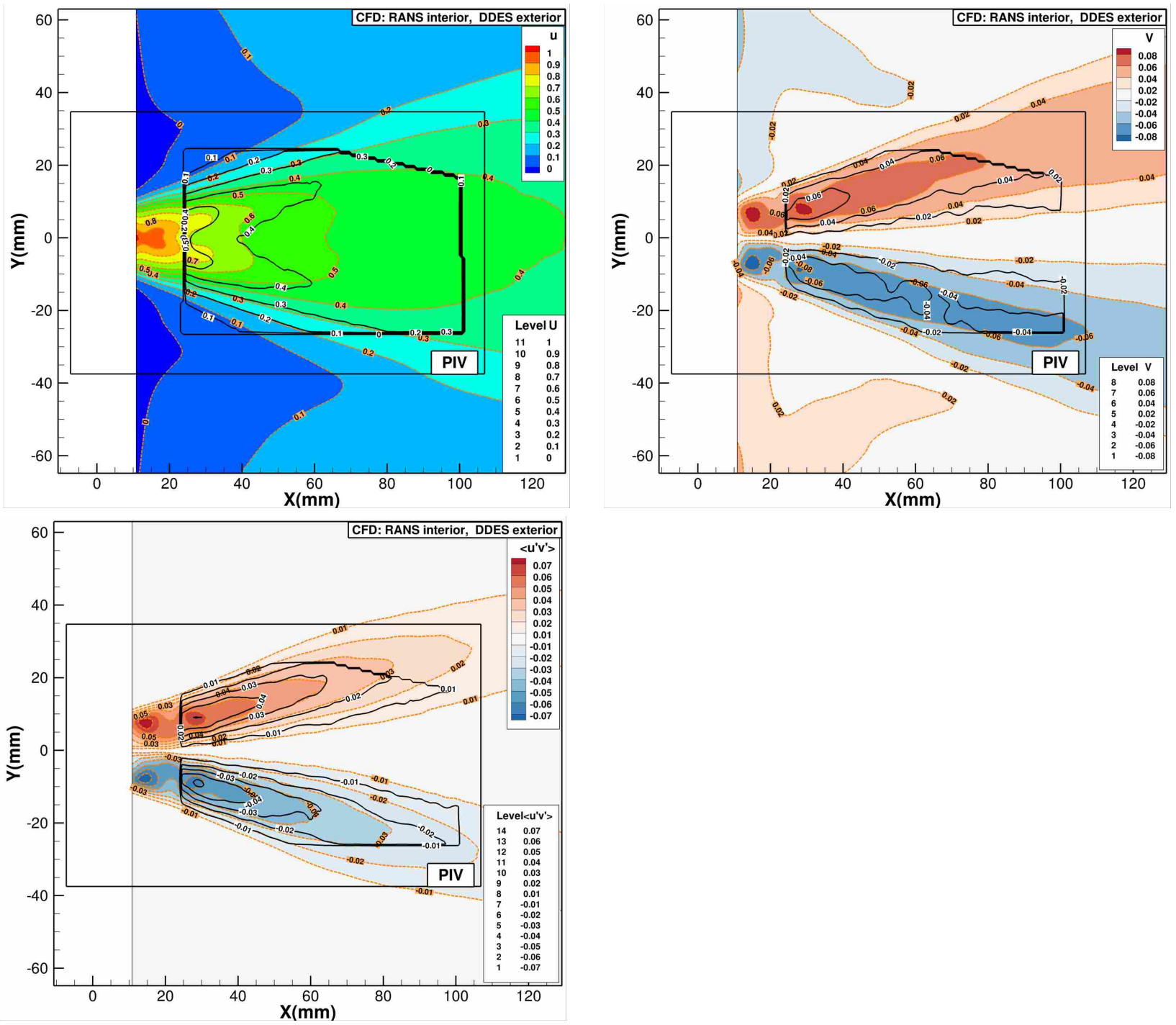

Figure 9: Comparison of simulation and PIV measurement of FML sweep jet. Plot origin $(0,0)$ is at center of jet nozzle. CFD is color flood contours, with orange lines and label. Heavy black lines inside "PIV" box are PIV measurement data. Plot legends indicate variables: mean U and V, and turbulent shear stress $\overline{u^{\prime} v^{\prime}}$. Velocities are normalized by sound speed, $335 \mathrm{~m} / \mathrm{s}$. (Truncated PIV contours on periphery are due to a limited field of view.)

planes recorded during the simulation. The CFD shear stress includes the resolved turbulence, plus the stress modeled using the strain rate and eddy viscosity.

Several features of the PIV data and CFD results are in good agreement, but there are also notable differences. The mean streamwise velocity $U$ exhibits good agreement at the outer edge of the jet, although the PIV data show the jet to more spread-out than the CFD, by a small amount. Both PIV and CFD exhibit two $U$ maxima in the jet's core region, which result from the the sweep-jet "pausing" briefly at maximum deflection, as indicated by the throat Kulite pressure signal. Again there are differences in the details of these two maxima, and significant differences in the velocity magnitude; at the minimum $x$ where there is PIV data, the CFD $U_{\max } \sim 0.75$, while for PIV $U_{\max } \sim 0.55$, so the PIV data are roughly $\sim 30 \%$ below the CFD results. The mean vertical velocity $V$ also shows good overall similarity in the shape of the jet, with the CFD results roughly $\sim 30 \%$ higher than the PIV data. The peaks in the $\overline{u^{\prime} v^{\prime}}$ shear stress, both positive and negative, tend to align with the region of maximum strain rate in the jet's mean flow, and thus indicate the spreading angle of the jet. Simulation and PIV agree very well on this spreading angle. The maximum magnitude of the shear stress, inside the PIV window, is $\left|\overline{u^{\prime} v^{\prime}}\right|_{\text {max }} \sim 0.06$ for CFD, compared 
to $\left|\overline{u^{\prime} v^{\prime}}\right|_{\max } \sim 0.05$ in the PIV data. Both CFD and PIV also exhibit small local peaks in the shear stress magnitude at $x \sim 27 \mathrm{~mm}$, which is spatially associated with the shock cells seen in Fig. 5.

In general, the CFD simulations capture the main features of this single sweep-jet flow very well, most importantly, the dominant frequency and spreading rate of the sweep jet. Pressure histories at the nozzle throat lead to the selection of SST-RANS modeling for the interior of the oscillator. The SST-DDES model is favored for the exterior flow based principally on the anticipation that separation from the rudder will largely determine the accuracy of the full-tail simulations. The results shown here suggest that DDES is suitable for the near-field external flow, but nothing yet can be stated about the far-downstream region, or about modeling accuracy in the presence of an adverse pressure gradient.

\section{Unsteady Sweep Jet Boundary Conditions}

The recorded history of the flow at the throat of the single sweep jet is used to create highly realistic sweepjet inflow boundary conditions for the external flow; this provides consistently reproducible jet behavior and greatly reduces computational cost, at a small penalty in reduced realism of the full simulation. The benefits and validity of the using unsteady nozzle-throat boundary conditions (BCs), and the processing steps used to create the BCs, are described in this section.

This "external-only" strategy for the full-tail simulations has three significant benefits. One benefit is to reduce computational cost. The single jet simulation was run using $\sim 18 M$ grid points for the oscillator interior, so 31 similar oscillators would have required $\sim 550 \mathrm{M}$ grid points. In comparison, the grid for the exterior-only simulation of the full tail has $\sim 220 M$ grid points. If all oscillators were included in the fulltail simulations, they would have accounted for $\sim 70 \%$ of the total grid points, with direct implications for computational cost. Using the unsteady throat BCs makes that part of the computational cost essentially zero. The second benefit is to provide control over the sweep jets. Both the experiment and single-jet simulations of the single jet had occasional random "hiccups," in which the throat pressure oscillation was much different than normal. While this may be physical, it could adversely affect a very long and expensive simulation. This behavior was avoided in selecting the jet history to supply the boundary conditions. Finally, the phase, frequency and strength of the sweep jets can be explicitly adjusted, within some limits, to model the external effects of slightly different oscillator conditions.

The validity of powering the external sweep jets with unsteady nozzle throat BCs is affected by several flow-physics and algorithmic considerations. This topic is addressed only briefly in the present work, as the focus has been on completing the full-tail simulations; it deserves further study to help advance this sweepjet flow control technology. A key flow-physics issue is whether the external flow can influence the internal flow. If the nozzle throat flow is choked, the oscillator interior flow is isolated from the exterior, and the nozzle-throat boundary conditions can be highly accurate. The single-jet simulations discussed here involve jet supply pressures of $\Delta p / p_{\infty}=2.585$ and $p_{\infty}=1$, exhausting to atmospheric or lower pressures, which yields choked mean flow. However, there are small near-wall regions of reversed flow at the nozzle throat, as seen in Fig. 5, even if the main flow is choked. In some applications, even lower pressure jets may be used, in which the external pressure clearly affects the interior flow. Thus, the relevant concern is the level of outside influence that flows upstream into the oscillator, as a function of the gross mean parameters at the nozzle, such as its pressure ratio. We do not here attempt to address issues that arise from mean subsonic flow at the nozzle throat. No problems were encountered using the nozzle-throat boundary conditions with choked mean flow.

The key steps in implementing the nozzle-throat BCs are summarized here, and some additional details are given below.

1. A single-jet simulation is run at approximately the correct pressure pressure ratio until it reaches its asymptotic unsteady state.

2. The flow history is recorded at the plane of the nozzle throat over many oscillation periods.

3. A time-interval is selected for the boundary conditions, consisting of a small integer number of oscillation periods, based on the signal's dominant native period. This is a $3 \mathrm{D}$ data set consisting of two space dimensions and time, containing all simulation variables. 
4. The flow in the selected time-interval is "regularized" to make it fully periodic and without reversed flow.

5. The flow is rotated to the orientation of the installed nozzles on the full tail.

6. Spatial interpolation is used to transfer the single-jet simulation data onto the grid points of the full-tail simulation, as the grids differ in the present work.

7. Minor scaling in the nozzle mass-flux and pressure have been applied in some cases, to model supply pressures and mass-flow rates different from the single-jet simulation.

8. A specialized time-dependent boundary-condition routine selects the appropriate time in the boundary data, based on physical time and sweep-jet frequency, and the phase of the individual sweep jet, and applies that data to the nozzle boundary plane in the CFD solution.

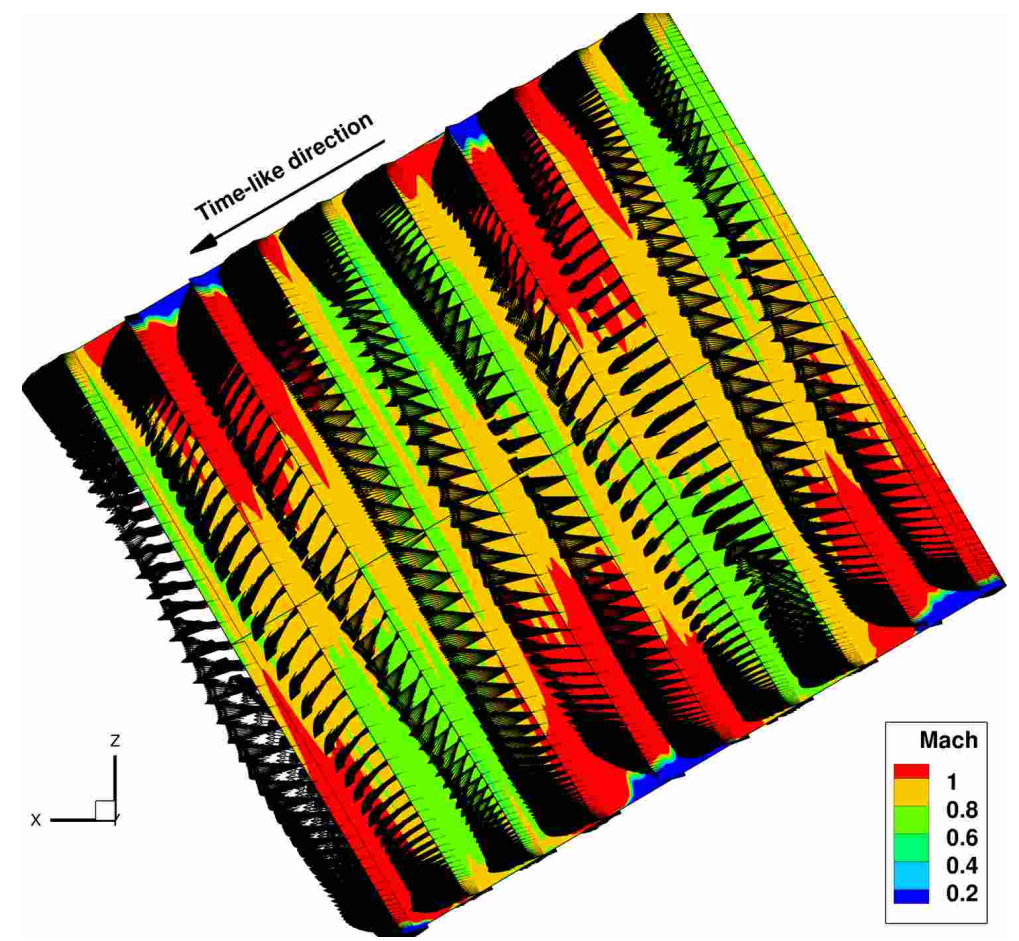

Figure 10: Visualization of the nozzle throat boundary condition data file, which includes two dominant periods in the time-like direction. Note that this is $x-y-z-t$ data rendered on an $x-z$ plot. Color flood contours of Mach number are given on the centerplane and at selected planes in time-like direction. Velocity vectors are visible above the centerplane and they illustrate the variation of the flow in the plane that becomes the instantaneous throat velocity field. The instantaneous boundary conditions are extracted on a plane perpendicular to the time-like direction, that advances in the time-like direction.

The nozzle-throat inflow BCs must be representative of the true flow and purely time periodic, so it can be repeated seamlessly and endlessly, for as long as needed for the exterior flow to reach statistical convergence. The native recorded nozzle throat history does not meet this requirement. The oscillator flow considered here has a strong dominant peak in its energy spectrum, but it also has significant energy outside that spectral peak. The first step in processing the native nozzle throat data is to find an integer number of periods, $N$, that has small non-periodicity. A search algorithm was constructed that measured the RMS difference in the solution and its first and second time derivatives at integer oscillation periods, in which the period was determined by Fourier analysis of the throat data, as in Fig. 6, over the full asymptotic solution history. An interval was selected with small native nonperiodicity in the RMS solution value and first derivative. Then, the non-periodicity is removed from this history. The native data are discontinuous at the ends of the $N$-period record. This discontinuity can be represented as a mean slope, which is subtracted from the data, which distributes the native non-periodicity over the full $N$-period record and gives $C^{0}$ periodicity. Increasing the number of cycles included in this record reduces this distributed error, at any point in time. In the small regions of instantaneous reversed flow, the velocity was set to zero, and the pressure and density were unchanged. The final step in achieving periodicity is to lightly smooth the flow variables with mixed 


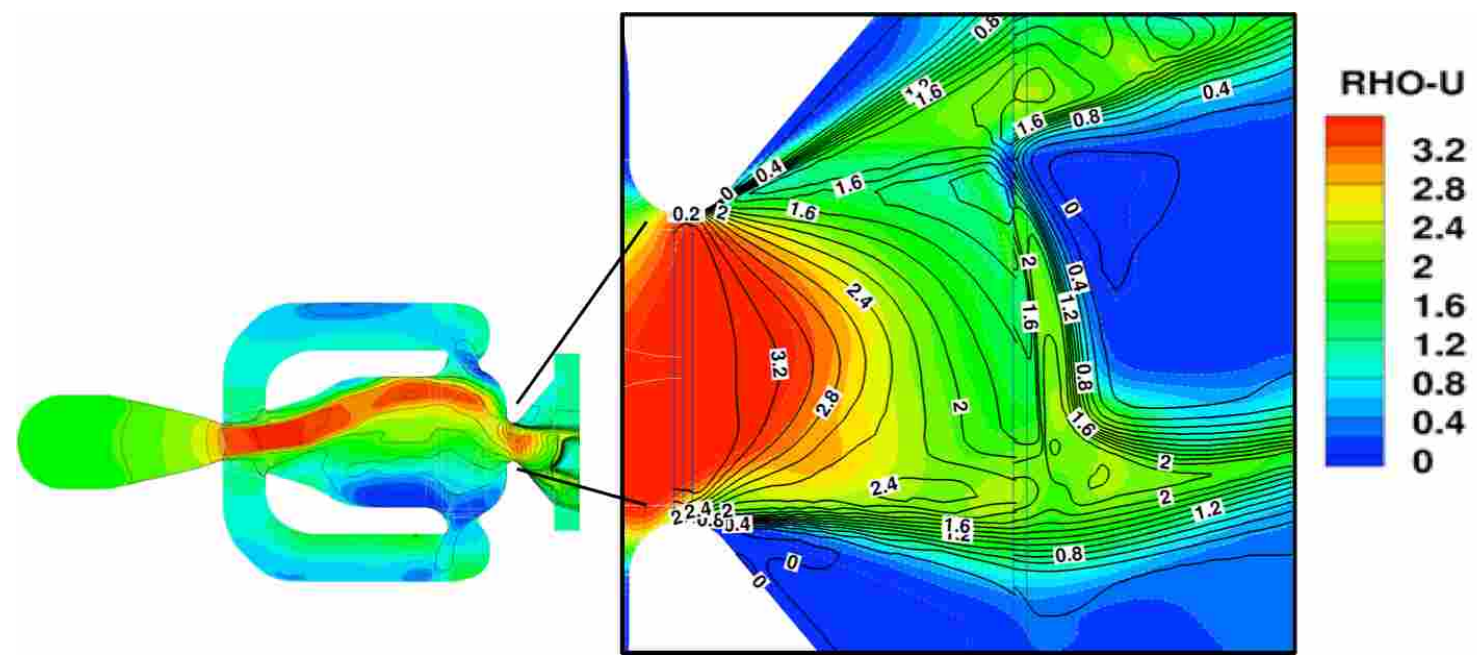

Figure 11: An overlay of contour plots of the streamwise momentum from the full interior-exterior sweep-jet solution (color contours) and from the exterior-only solution powered by throat boundary conditions (black line contours) demonstrate goods agreement downstream of the nozzle throat.

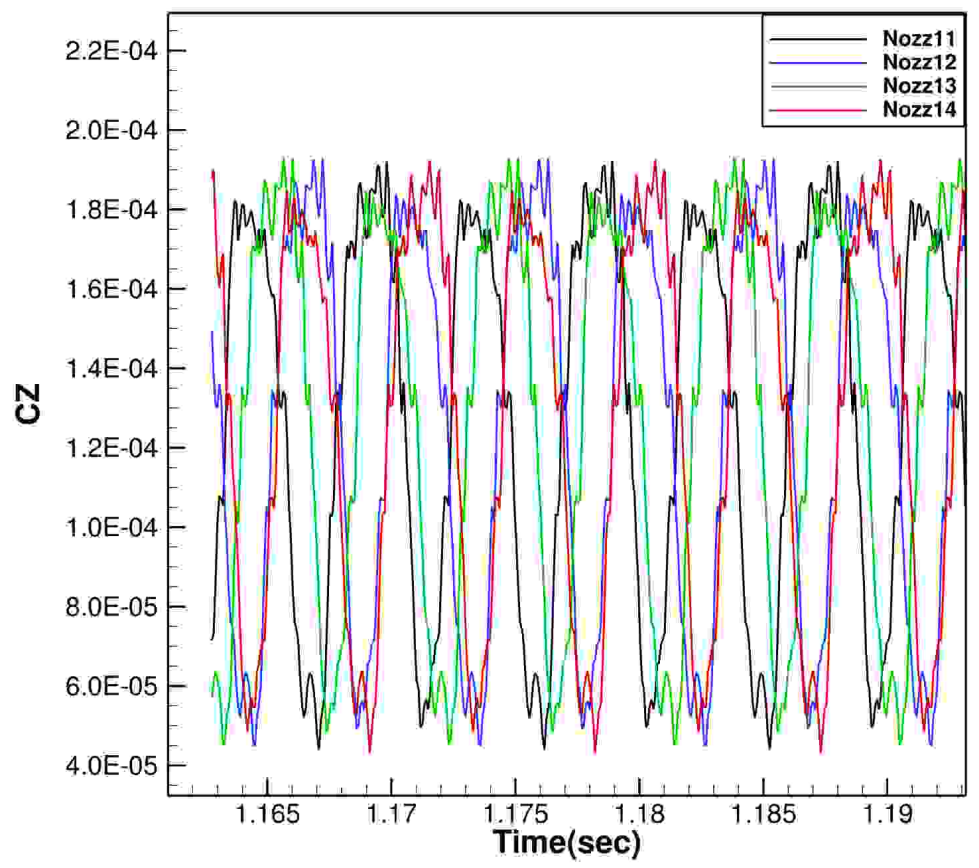

Figure 12: The vertical momentum flux coefficient, CZ, of 4 adjacent nozzles, numbered 11 through 14, in a full-tail simulation powered by sweep-jet boundary conditions.

second and fourth-order smoothing that respects the periodicity in the time-like direction. The rotation and interpolation onto the orientation and grid spacing of the sweep-jet nozzle of the full-tail grid system are mathematically straightforward.

The result of this processing is a 3D data set, of two space dimensions and time, representing a time interval that is an integer multiple of the dominant oscillator period. A graphic representation of the $\mathrm{BC}$ data file used by Overflow for the full-tail simulations is given in Fig. 10. For this illustration, the time-like direction has been converted into an artificial space dimension, normal to the plane of the nozzle, which is oriented as in the full-tail simulations. Two dominant periods of the jet oscillation are present in the data, as seen by the two large patches of high Mach number flow on the upper-left edge of the Figure. There are two dominant patches of low and high Mach number flow on both the upper-left and lower-right edges, when one 
properly accounts for the periodic boundaries at the lower-left and upper-right edges of the image. Significant cycle-to-cycle differences in the details of the contours can be seen in the two cycles.

The ability to alter the available nozzle throat boundary condition data to match a specific operating condition enhances the flexibility and utility of this jet BC concept. The BC's equivalent supply pressure, mass flow and frequency can all be modified from the original oscillator simulation. In the present work, the mass flow and pressure have been adjusted by as much as $\sim 18 \%$, by scaling the density and pressure, while preserving the temperature, velocity and Mach number of the flow. The dominant jet frequency was set to $220 \mathrm{~Hz}$, which was the frequency observed in the oscillator simulation when the full-tail simulations were started. The details of BC scaling have not been studied here. If sweep jets mature into a useful flow-control device, study of this topic is warranted.

The data in Fig. 10 are read and processed by a newly-developed Overflow BC subroutine. The subroutine reads the boundary-condition file once at the start of a run, and receives three input parameters during the course of a run: the "physical" time, the physical time-period (inverse frequency) over which to replay the boundary-condition file, and a phase shift. (Of these, only the current time is updated in the present simulations.) This is sufficient information to determine the correct time-plane in the boundary-condition data set, which is extracted using third-order Lagrange interpolation in the time-direction. The solution data are imposed at the boundary grid points without consideration of the mathematical characteristic of inflow and outflow. This is reasonable for choked flow, but not for lower-speed flows. Based on discussions with personnel with extensive experience testing sweep-jet flows, the relative phase of each of the 31 jets was set randomly. The frequency was set to be similar in all jets, so there is no "beats" phenomenon, in which jets move slowly through differing phase relations producing a low frequency signal envelope. A subtlety related to Overflow is that the nozzle throat grids on which the boundary conditions were applied was made small enough that Overflow's process of grid-splitting for load-balancing left this grid whole; this enabled simple programming of the boundary condition software.

Two validation tests were applied to the nozzle-throat BCs. Early in the work, the basic concept was tested. Two RANS simulations of a single jet were compared: one included the full interior and exterior flow, while the other was just the external flow, powered by the nozzle-throat BCs. The processing needed to obtain a smoothly periodic solution was performed, but the jet pressure and mass flow were not altered in this test. Fig. 11 gives an overlay of contours of the streamwise momentum, and good agreement between the two sets of contours is seen. (The jet operating conditions in this test differ from those used in the $\Delta p / p_{\infty}=2.585$ and $p_{\infty}=1$ FML test, and thus the jet flow fields also differ.) Another validation test is to observe the history of the nozzle's vertical momentum flux coefficient, $C Z$, in a full-tail simulation, which is given in Fig. 12. The installed jets oscillate principally in the $x$ - $z$ plane, and the $C Z$ indicates the jets' flow angles. The $C Z$ data are products of Overflow's loads integration routines, and they reflect what the CFD simulation is actually experiencing. $C Z$ is a load applied to the body, so these momentum fluxes indicate fluctuating lift on the tail. The period of the signals matches that which was requested, $220 \mathrm{~Hz}^{-1}$. The relative phase differences between signals are not regular, consistent with the input specification of random phases. The exact periodicity observed over two cycles is also as intended. Thus, the boundary condition process is functioning as intended; the external jet created by the boundary conditions is similar to the full interior-exterior simulation, and the integrated momentum fluxes have the appropriate frequency and phase.

\section{Full Tail with 31 Sweep-Jets}

A primary goal of the Project's full-tail experiment was to demonstrate that the target for improved maximum loading on the tail can be achieved, and hence that sweep-jets are an effective flow control technology to retard boundary layer separation, at flight conditions. The goal of the CFD work is principally to replicate the experimentally observed aerodynamics. Because this flow is aerodynamically complicated, achieving good agreement with experiment is viewed as a significant challenge. CFD also has the ability to provide a detailed picture of the fluid dynamics of the sweep jets. The primary challenges in the full-tail CFD simulations are the: (1) range of relevant length scales, and the associated size and complexity of the grid system, (2) boundary conditions that provide the sweep jets, described above, (3) large ratio of time scale, 
from the time step needed to resolve the sweep jets to the overall time needed for flow convergence, and (4) turbulence modeling for the sweep jets.

At this point in the paper, specific values of mass flow, supply pressure and aerodynamic behavior are replaced by omission, increments or non-specific terms like 'low' and 'high.' On graphs, only the 'zero' tick mark is true.

The flight conditions for all results presented here are a freestream Mach number of $M=0.15$, a yaw angle of $\beta=0^{\circ}$, and a Reynolds number of Rey $=1.05 \mathrm{M}$ per foot. Simulation results are given for three rudder deflections, $\delta_{r}=20^{\circ}, 25^{\circ}$, and $30^{\circ}$, while experimental data also include $\delta_{r}=0^{\circ}$ and $15^{\circ}$. SST-RANS and SST-DDES simulations were run. Simulations were also performed at $\beta=5^{\circ}$, but those results are not presented here.

\section{VI.A. Geometry \& Grids}

The experimental test article is a Boeing 757 tail, obtained from an Arizona aircraft boneyard, and modified to include sweep jets. Figure 13 shows the tail installed in the tunnel. The 37 sweep jet oscillators and nozzles are in a housing strip that is roughly $1 / 2^{\prime \prime}$ thick, installed just upstream of the rudder hinge line on the starboard side of the tail. The housing strip alters the native Boeing tail's shape and its aerodynamics, and its effect on the surface pressure is discussed below. The tail is mounted on a teardrop-shaped blister which is attached to the floor of the Air Force NFAC $40^{\prime} \times 80^{\prime}$ wind tunnel at NASA Ames Research Center. The blister elevates the root of the tail above the tunnel-floor boundary layer. The experiment is described in the papers by Andino ${ }^{3}$ and Whalen. ${ }^{21}$ The experiment measured distributed pressures and integrated loads on the tail over a wide range of operating conditions. The performance was observed to be very good at several operating conditions, including one with only the bottom 31 jets blowing, of the 37 installed. This case was selected for CFD simulations. The simulations have the lowest 31 jets blowing. All nozzles are present in the grid; the lower 31 jets are powered, while the upper 6 nozzles have a no-flow-through boundary condition across the throat.

Several details are relevant to the comparison of experiment and CFD. The tail is $\sim 75 \%$ of the height of the $40^{\prime} \times 80^{\prime}$ wind tunnel, and significant tunnel interference effects are expected. The wind tunnel walls are included in the simulations, but their geometry was not exactly preserved. The entire tunnel was approximated as having a constant cross-sectional area. The walls used in lieu of the tunnel's inflow bellmouth and outflow diffuser are treated as slip walls. The starting point of the no-slip wall was adjusted to match the measured ${ }^{13}$ boundary layer thickness of $\delta_{99} \simeq 12^{\prime \prime}$ in an empty tunnel, a short distance ahead of the tail's location. To account for the acceleration due to boundary layer growth in the test section, the normalized pressure and load coefficients were computed using the estimated static and dynamic pressures at the tail's location, excluding the tail's effects on the freestream. This is an approximation, as it neglects some additional change in the "local freestream" due to the tail's blockage effects. The true wind tunnel walls diverge to accommodate normal boundary layer growth, and tunnel data are also corrected with the goal of minimizing tunnel effects.

The tail's interior is vented to the mounting blister interior, which then vents to both the tunnel interior and to atmospheric pressure surrounding the test section. "Brush seals" are used to limit flow through the junctions between the tail, blister and tunnel floor. Pressures on the tail's interior surfaces contribute to integrated loads. The interior pressure is estimated as the average pressure around the perimeter of the tail-blister junction, and then applied to the tail-root area and included in CFD loads calculation. The interior pressure principally affects the lift and drag, but not the side force. The experimental and CFD drag forces on the tail also includes the sweep jet thrust, which is reported as negative at some experimental operating conditions. The experimental data tables suggest that the uncertainty in yaw is $\beta \pm 0.5^{\circ}$, and the uncertainty in the rudder angle is $<0.01^{\circ}$. CFD runs were at nominal values, $\beta=0^{\circ}, 5^{\circ}$, not at the actual values. The rudder leading edge has "cut-outs" to provide hinge clearance at high rudder deflection; the cut-outs were covered with tape in some runs, and left open in others. The CFD geometry modeled neither the rudder mechanism nor the cutouts, and comparisons are with test data with covered cutouts.

The CFD grid system used in this work represents a balance between finite computational resources and the need for good spatial resolution of important flow features. The largest full-tail grid system has 575 

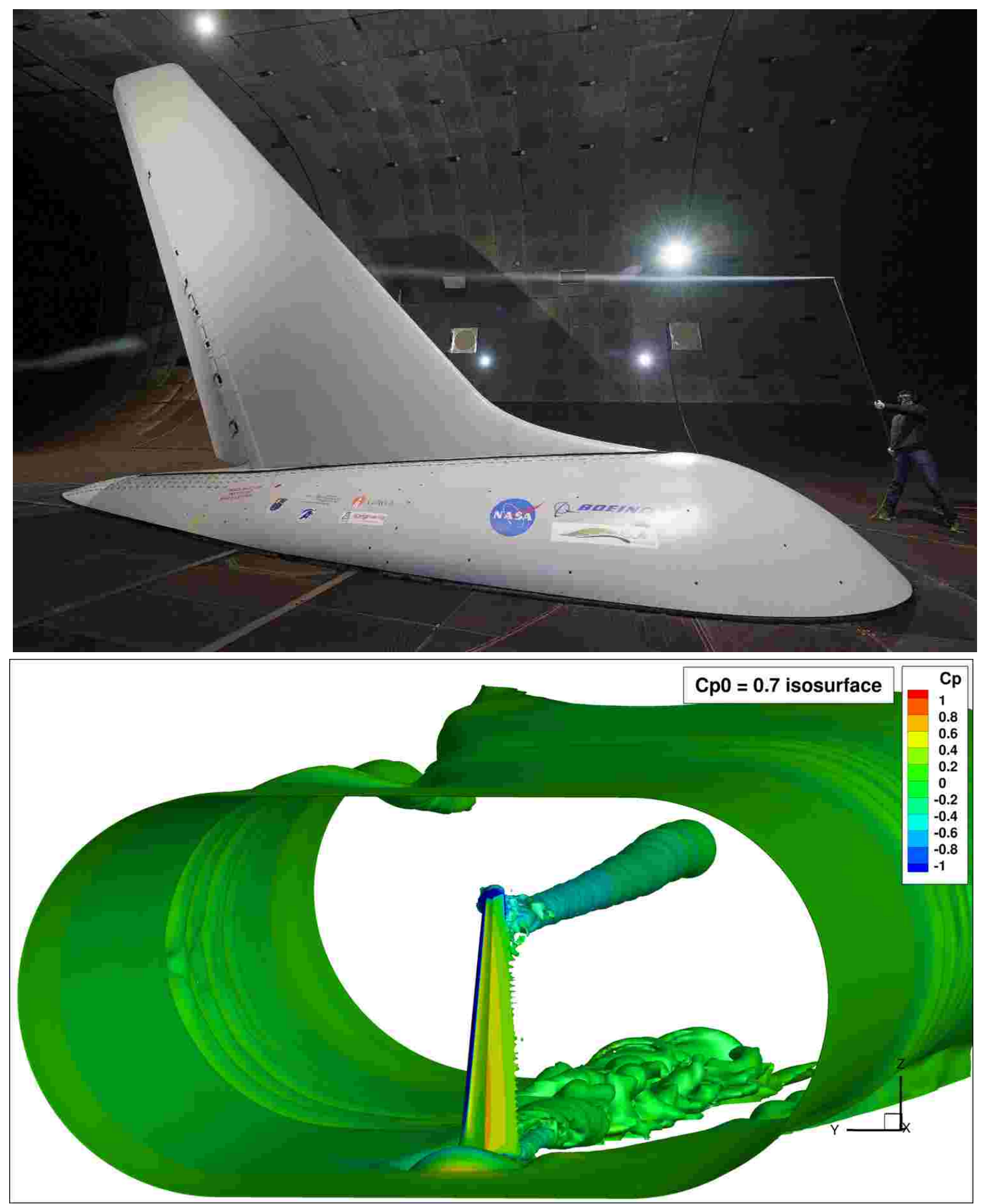

Figure 13: Full tail installed in NFAC $40^{\prime} \times 80^{\prime}$ wind tunnel. Smoke flow-visualization testing (top). CFD isosurface (bottom) of stagnation pressure coefficient, $C p_{0}=0.7$, colored by $C p$. Key features that are visible include: the tail with a deflected rudder on its mounting blister; tail tip, rudder root, and blister horseshoe vortices; agglomeration of tunnel boundary layer fluid due to tail-induced circulation; small short "streamers" on the rudder trailing edge, from non-uniform boundary layer thickness on rudder with active sweep jets, flowing off rudder and into wake. 


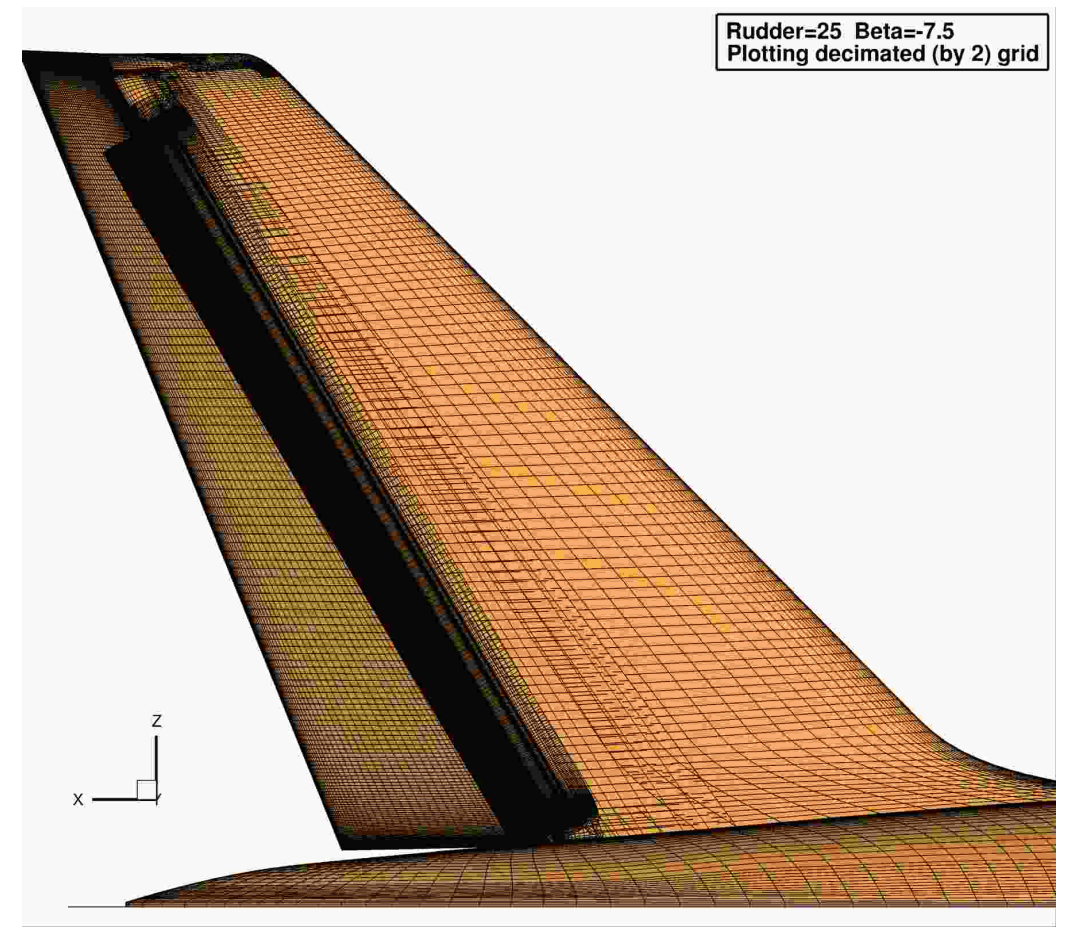

Figure 14: Surface grid on the tail, showing every second grid line. The dark band on the front section of the rudder is the intermediate-field grid, intended to resolve the near- to intermediate-field of the sweep jets.
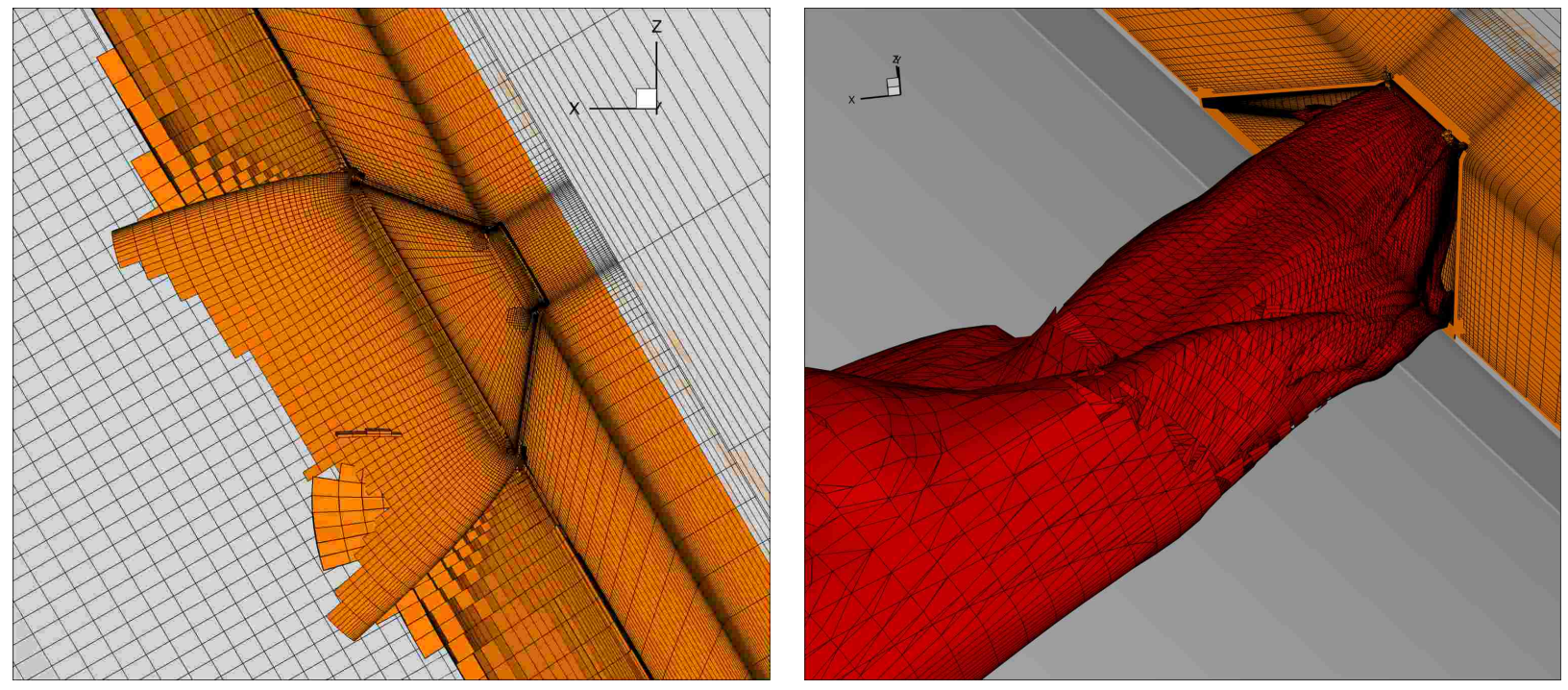

Figure 15: Grid for a single sweep-jet nozzle (left) with the 14 grids associated with each nozzle highlighted in orange. A $M=0.4$ isosurface of sweep jet highlighted in red (right), with full-resolution grid spacing.

individual block grids, with 518 grids devoted to the 37 jet nozzles, and $216 \mathrm{M}$ total grid points. The rudder deflection affects grid overlapping and overset connectivity, and a few extra grids were needed at the highest rudder deflection, $\delta_{r}=30^{\circ}$. The primary region of interest is downstream of the sweep-jet nozzles and on the rudder, but other regions must be well resolved to enable a clear assessment of the sweep-jet simulation. Figure 14 gives an image of the surface grid, decimated by 2 in both surface coordinate directions. An "intermediate-field" grid was designed to cover the sweep jets through an expected region of strong adverse pressure gradient on the forward part of the rudder, and it appears as a dark band downstream of the rudder hinge line. The sweep jets oscillate in the vertical direction, and the entire (suction-side) rudder boundary layer may be affected by jet-induced eddies. The intermediate-field grid spacing is uniform at 
$\Delta \zeta=0.1^{\prime \prime}$ where $\zeta$ is "up" the rudder; this grid has $3045 \times 71 \times 96$ points in the directions up the rudder, quasi-streamwise, and normal to the wall. The rudder grid aft of the intermediate-field grid was not highly refined, as part of the compromise between computational cost and accuracy.

It is essential to recognize what grid spacing is achieved on flow features relevant to sweep jet performance. The grid resolution of the near-field sweep jet is shown in Fig. 15. The grids highlighted in orange are associated with each nozzle, and are repeated at all 37 nozzles. The gray grids are associated with the tail and rudder as a whole; for example, the intermediate-field grid is seen in the left side of the left image. Only the active grid points, as selected by the Pegasus overset grid-connectivity software, are shown (although the plotting routine is not fully precise in rendering this distinction.) The nozzle throat has $69 \times 91$ grid points, in contrast to the $201 \times 291$ grid points at the nozzle throat used for the single sweep-jet simulations. The grids lines overlaid on the $M=0.4$ Mach number isosurface of a sweep jet, in the right image, is a straightforward and relatively meaningful image of grid resolution. Counting grid lines (and we rotated the image to see the full jet) reveals that the near-field grids have $\sim 25$ grid points across the width of the jet, and the intermediate-field grid has $\sim 16$ points across the vertical dimension of the jet at this location. The choice of the $M=0.4$ isosurface is somewhat arbitrary, but reasonable; it slightly exceeds the local Mach number of the non-jet flow at $M \sim 0.3$, and thus is close to what we think of as the "outer edge" of the jet.

A critical assessment of the current grid resolution must conclude that some regions of the flow are significantly under-resolved. The $\sim 16$ grid points across the jet, in combination with the fifth-order spatial discretization, is probably "borderline adequate" to provide weak algorithmic diffusion in the momentum convective terms, if the jet had a smooth fully-developed velocity profile. However, as seen in the single-jet results in Fig. 5, the near-field jet has a potential core surrounded by a shear layer; that shear layer cannot be well resolved on this grid. Even less well resolved are the turbulence variables of turbulence models with transport equations. Across a simple shear layer, typically, the momentum variables "ramp up" from one side to the other, while the turbulence variables reach a local maximum value near the middle, so they ramp-up and ramp-down. Therefore, the turbulence model requires roughly twice the number of grid points that are needed to resolve the Navier-Stokes variables. This simulation is very likely affected by cancellation of errors in some regions: the modeled turbulence is under-resolved and therefore artificially weak, but these effects are partially offset by moderate algorithmic diffusion of the jet's momentum field.

\section{VI.B. Simulation \& Processing Details}

Details of how the full-tail simulations were run and processed are summarized here. The full-tail sweep-jet simulations were run using a "bootstrap" strategy that involved starting from initial conditions that were close to the final state. In LES, the computational cost scales linearly on the ratio of physical convergence time to time step. Thus, reducing the physical time needed to converge the flow is one means of reducing $\mathrm{CPU}$ costs. The total physical time needed for a flow to reach its asymptotic state cannot be accurately anticipated in most cases, but the convection of the loads-induced circulation sets a lower limit on that time. The starting solutions were obtained using steady jets, in lieu of the unsteady sweep jets, run with the same mean mass flux as the sweep jets. Steady jet cases achieved side-force coefficients that were often only a few percent below the sweep-jet results, but they could be run at time steps which were $O(100)$ times larger than the LES time step and would converge in $O\left(10^{3}\right)$ time steps. When the sweep-jets were "switched on," the steady jet flow field is replaced by the sweep-jet field in some small multiple of the flow-through time period, for flow over the rudder. Convergence after starting the sweep jets involves, in these cases, a relatively small change in the global circulation field, and convergence to some tolerance is faster than starting the sweep-jet simulation from crude initial conditions. There are potential deficiencies in this bootstrap approach, for example, adverse effects of hysteresis and non-uniqueness, and the probability that the strategy for setting initial conditions yields a poor approximation of the final state, at some operating conditions.

An example of a simulation, run as described above and with DDES turbulence modeling, is presented in the history of the side force coefficient $C Y$ in Fig. 16. This history starts at iteration iter $\sim 1500$, after the steady-jet steady-state RANS simulation was almost converged. The side force, $C Y$, without tunnel corrections starts at $\sim 97 \%$ of its asymptotic value. The rudder flow-through time period is roughly $0.05 \mathrm{~s}$, and much of the increment in rudder side force becomes established in $0.1 s$, after switching the nozzle 


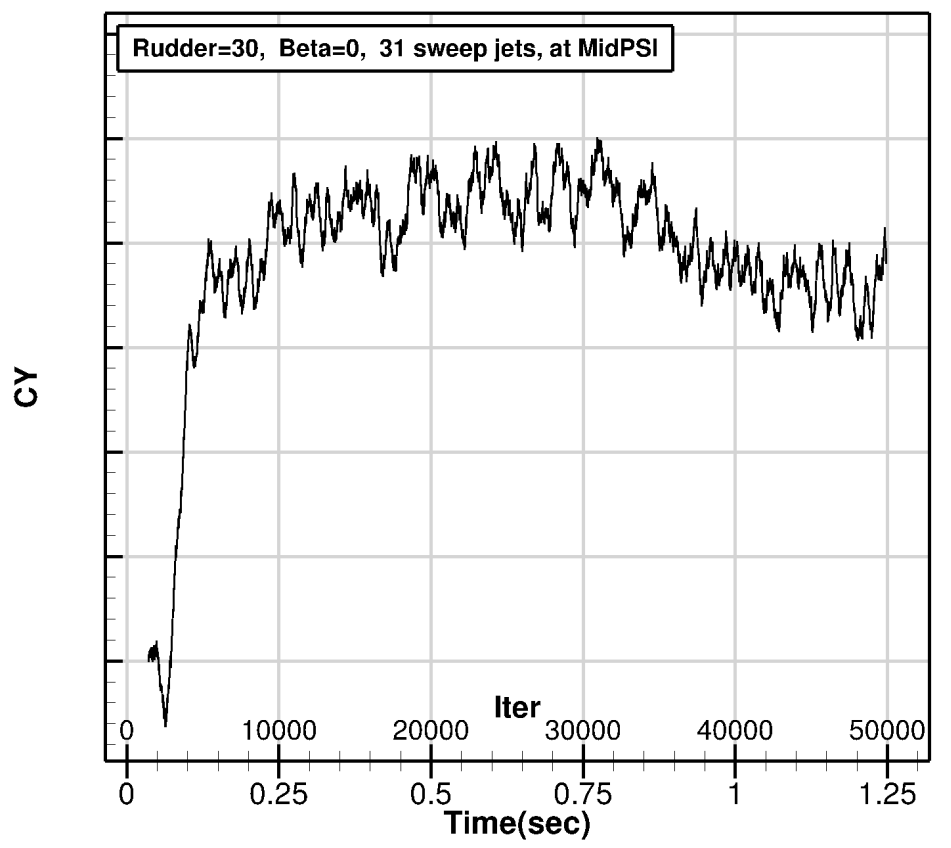

Figure 16: History of the native Overflow load history, after restarting from a solution run with steady jets in lieu of sweep jets.

boundary conditions from to steady jets to sweep-jets. This simulation appears poised to move to a slightly higher $C Y$, were the simulation to be continued. Typical of our general experience in D/DES simulations, some degree of convergence uncertainty must be accepted in the results. The averaged integrated loads displayed in subsequent figures are obtained from long-time averages, which in this case was from iter $=27 k$ to the end of the simulation. Loads are then corrected for the freestream at the tail's location. This simulation was run for 894 hours on 800 Ivy Bridge cores for a total of $\sim 715 k$ CPU-hours.

The time step used to compute the oscillator flow, $5 \mu \mathrm{s}$, is smaller than needed for the full-tail simulation which uses coarser grid spacing and cannot resolve such small length and time scales. The time step was set to $25 \mu \mathrm{s}$, for the full-tail simulations, and the effects of time step on accuracy were not evaluated.

\section{VI.C. Mean-Flow Simulation Results}

CFD results are presented for several simulations of the Boeing 757 tail, with and without sweep-jets powered in the lowest 31 of the 37 installed nozzles. These are time-averaged results, computed as described above from the asymptotic state of the unsteady simulations. All results presented are for a nominal yaw angle of $\beta=0$. Figure 17 shows that the flow is separated at the top 6 sweep-jet nozzles which are unpowered, and it is attached at all lower sweep jets which are powered. There are also pockets of low-speed flow near the trailing edge, and between the jets in the upper-most 7 of the powered jets, which may indicate local incipient separation. Near the base of the tail, the rudder's streamwise length is $l \sim 80^{\prime \prime}$ compared to the jet's initial dimensions of $0.5^{\prime \prime} \times 0.25^{\prime \prime}$, for a geometric mean dimension of $d=0.35$. Thus, the sweep jet's influence in the boundary layer persists to $l / d>200$.

Figure 18 gives the three most important integrated loads: side force, drag force, and yawing moment about the mean quarter chord, in the body axis, denoted by $C Y B A, C D B A$ and $C N B A 25$. These plots include experimental results at four power settings as characterized by the differential pressure, psid, between the sweep-jet supply and ambient pressures: off (NoPSID), LoPSID, MidPSID and HiPSID. The experimental data points are at rudder deflection angles of $\delta_{r}=0^{\circ}, 15^{\circ}, 20^{\circ}, 25^{\circ}$ and $30^{\circ}$. The jets-off data exhibits the onset of stall as $\delta_{r}$ increases to $30^{\circ}$. Increasing the jet's supply pressure from NoPSID to LoPSID and MidPSID gives a monotonic increase in the magnitude of $C Y B A$ and $C N B A 25$ across all $\delta_{r}$; the further increase to HiPSID gives a small further increase except at $\delta_{r}=25^{\circ}$. 


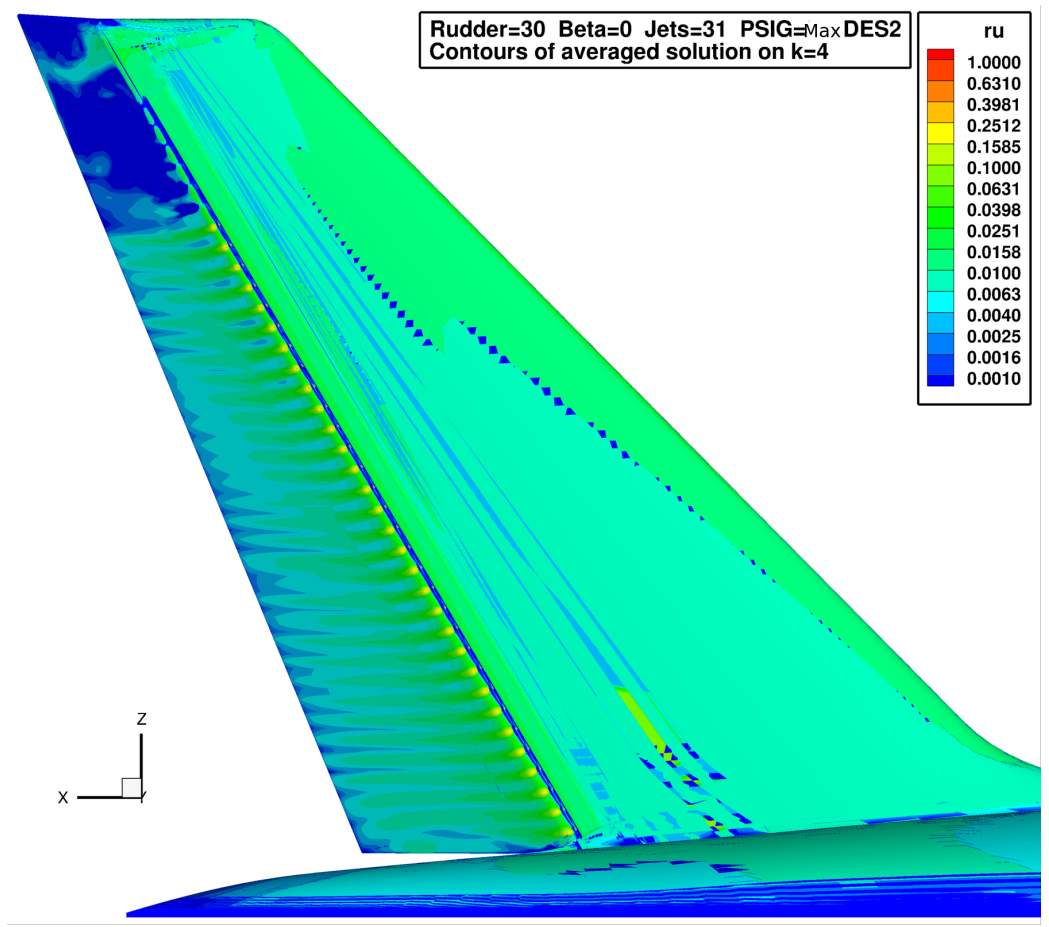

Figure 17: Time-averaged streamwise momentum $(\rho u)$ in the boundary layer just above the surface, from simulation using DDES turbulence modeling. Dark blue indicates reversed flow. The individual sweep jets are visible. There is incipient flow reversal near the trailing edge of lower rudder, shown by thin blue wedges, and full separation on upper rudder, where the uppermost 6 sweep-jets are not powered. (The blue 'stripes' and 'diamonds' on the tail are IBLANK-related plotting anomalies.)

The CFD results are in close agreement with the appropriate experimental data. CFD simulations were run with MidPSID and MaxPSID sweep jets. The MaxPSID sweep jet boundary conditions are from the single-jet simulation, and unscaled; the HiPSID BCs are the MaxPSID BCs with pressure and density scaled down to the lower supply pressure. Results for SST-RANS and SST-DDES turbulence modeling are included, but little difference is seen between these models, in these results. For the jets-off results, the CFD side force is slightly higher than the measured load, with the difference increasing to $\sim 0.04$ at $\delta_{r}=30^{\circ}$. The drag and yawing moments are similar in the CFD and experiment. For powered sweep jets, the side force is slightly overpredicted, but by a smaller amount than seen in the jets-off cases. The predicted yawing moment agrees well with experiment at all conditions. The tail's drag coefficient was computed in Overflow by integrating the tail's exposed surfaces, but excluding the area of the sweep-jet nozzle. The experimental loads are from a balance, and it includes the sweep-jet thrust. Thus, the simulation's aggregate nozzle momentum flux $C X$ is included in the drag results plotted here, to achieve similarity between experiment and CFD.

The surface pressure coefficient, $C p$, results are given in Figs. 19 and 20. The first of these Figures illustrates the pressure tap locations, which are arrayed along horizontal and quasi-vertical lines. The lowest three of the horizontal lines, at fixed vertical heights, VA, VB and VC, are within the lowest 31 sweep jets which are powered, at this operating condition; the top row, VD, is within the top 6 jet nozzles that are unpowered. The flow conditions for the results given in Fig. 20 are $\beta=0$, jet supply pressure MidPSID and rudder deflection $\delta_{r}=30^{\circ}$. Results for DDES and RANS turbulence modeling are given.

Several features of the $C p$ distributions are noted. The flow is from left to right, and the sweep jets affect the suction side of the rudder, which is the upper curve on the right side of each plot. There are typically four suction spikes on the suction side: (1) the tail's leading edge, the (2) leading and then (3) trailing corners on the housing which encloses the sweep-jet oscillators, and finally (4) the rudder's curved leading edge, over which the sweep jets flow. The rudder hinge line is close to the right-most of the suction spikes. On the pressure side of the tail, there is a broad pressure maximum at the rudder hinge line. A cluster of 

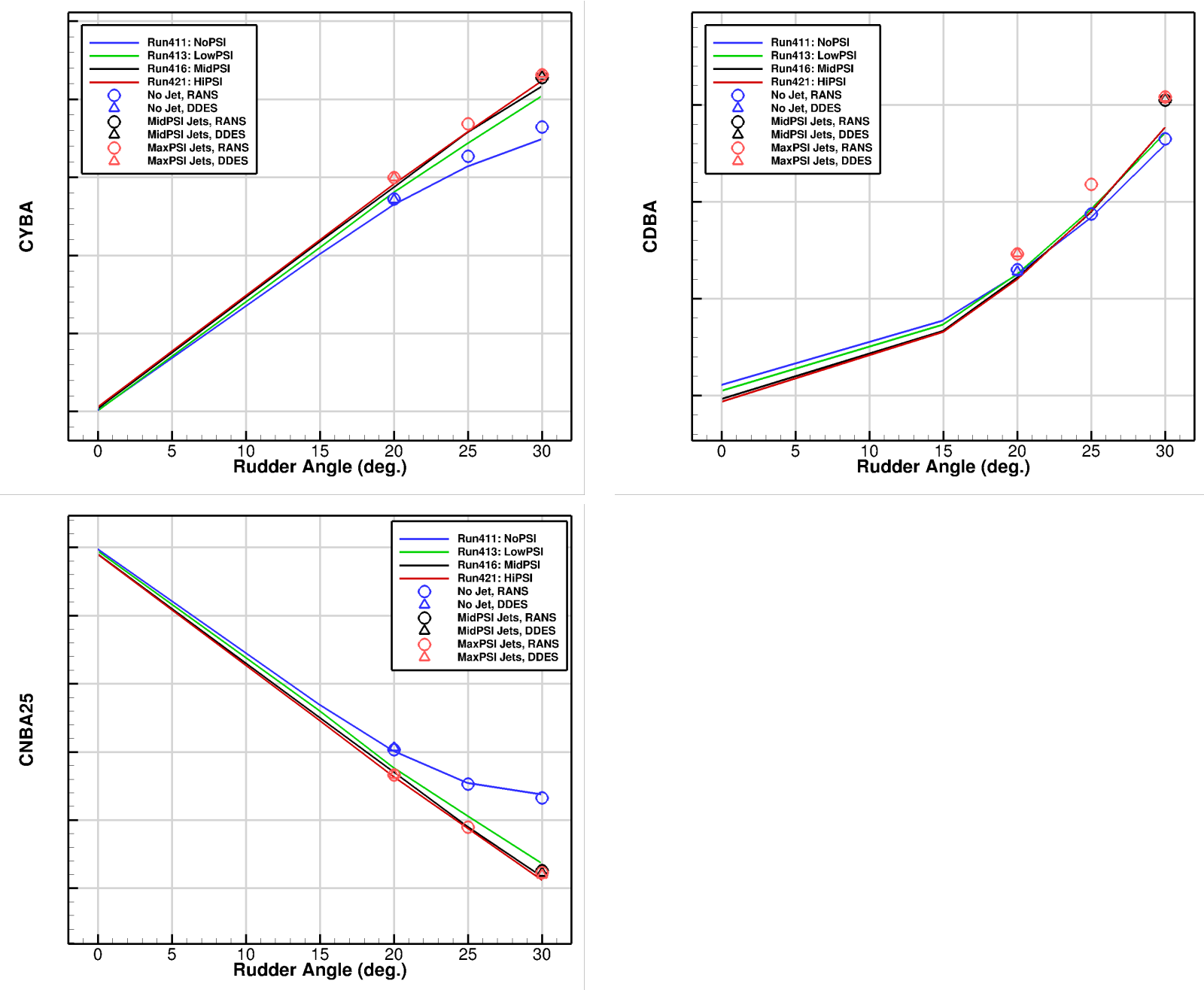

Figure 18: Time-averaged integrated load coefficients: side force, drag, and yawing moment as functions of rudder deflection angle. At operating conditions $M=0.15, \beta=0$. All CDBA data, experiment and CFD, include sweep-jet thrust.
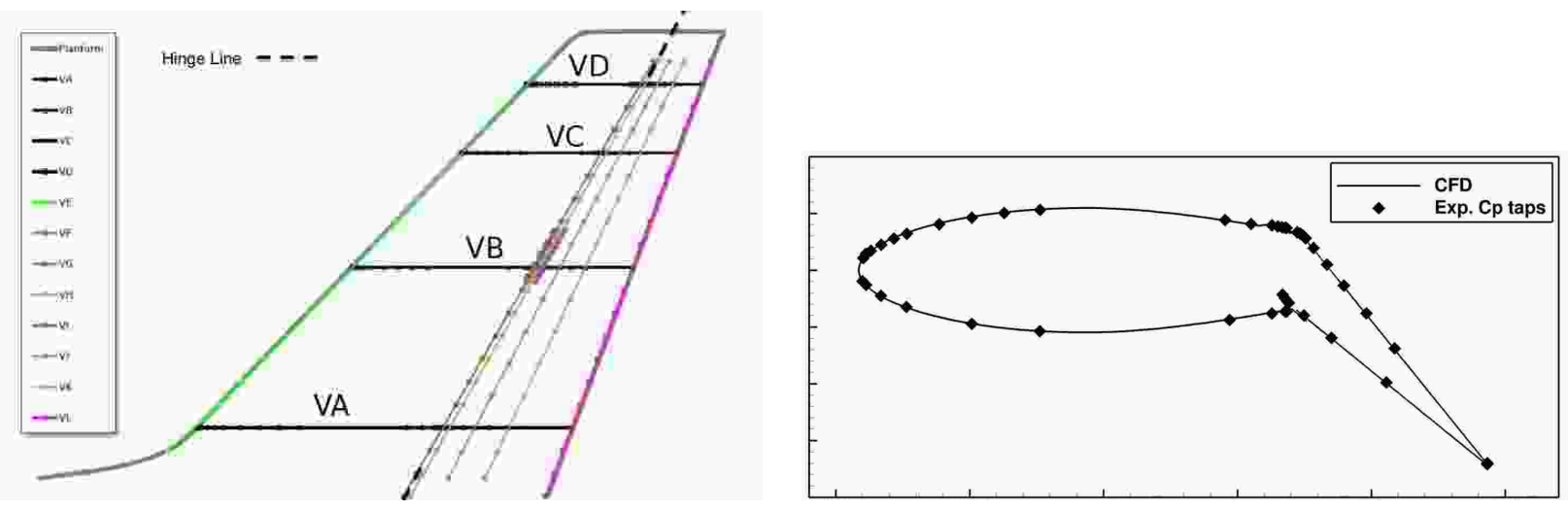

Figure 19: Pressure tap locations: the horizontal tap rows, the lowest VA to the highest VD. Individual pressure tap locations in the VA plane plotted with the CFD surface (not to scale). Note the interior taps, resulting from rudder deflection.

pressure taps reporting $C p \sim 0$ near the rudder hinge line are within the cavity of the rudder hinge, and do not measure the exterior pressure. The RANS and DDES CFD results are very similar except for the top 

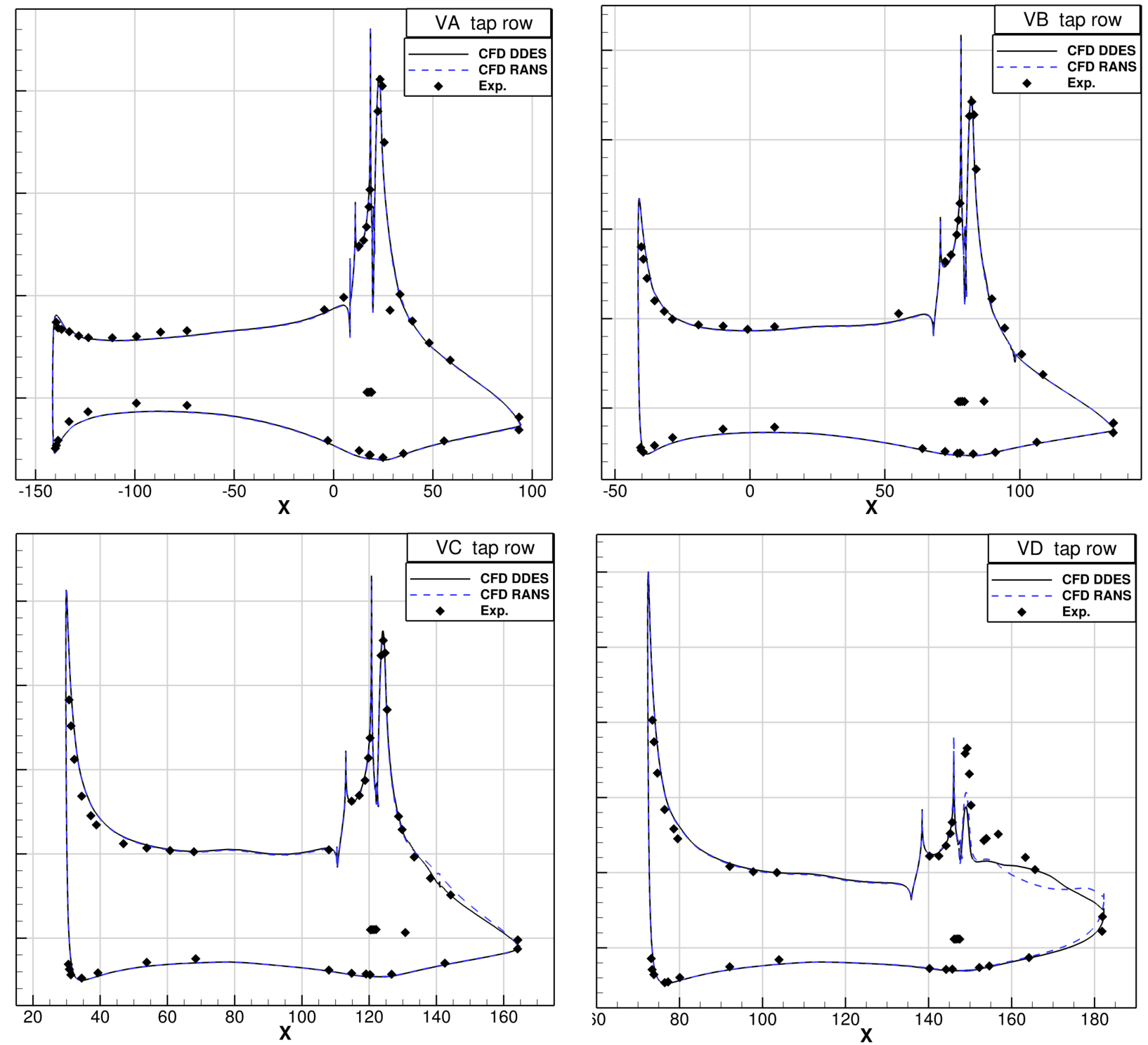

Figure 20: Surface pressure coefficient, $C p$, at the four horizontal (streamwise) tap rows: VA, VB, VC, and VD. Operating at $M=0.15, \beta=0$, sweep jet supply pressure MidPSID, and rudder deflection $\delta_{r}=30^{\circ}$. Results for SST-RANS and SST-DDES turbulence modeling.

pressure row, VD, where the flow is separated.

The agreement between CFD and experiment pressure distributions is excellent at most locations within the lowest three measurement stations, VA - VC, where the sweep jets are powered. On the rudder, the difference between experiment and CFD is consistently $\Delta C p<0.1$ and typically even smaller. The largest discrepancies in $C p$ are seen on the pressure side of the fixed tail at the VA station and on the suction side of the fixed tail at the VB and VC stations. This pattern of discrepancies with experiment is frustrating, as the largest differences occur in regions that ought to be simulated well by a Navier-Stokes solver. The discrepancy here implies that some "global" difference between experiment and CFD exists, and then, that the minimum uncertainty in the $C p$ is larger than the observed discrepancy on the rudder. The apparent high accuracy in predicted $C p$ on the rudder should be viewed as somewhat fortuitous.

The largest differences between CFD and experiment $C p$ 's occur in the separated flow at the VD station, where the sweep jets are not powered, and where the flow is separated. Ironically, in this simulation, separated flow is a greater challenge to accuracy than the sweep jets. 
The differences between RANS and DDES results are negligible at the VA and VB stations, and only become visible at the VC and VD stations. This result must be interpreted cautiously. The streamwise grid spacing dominates the DDES switching between RANS and LES modes, and that grid spacing decreases with increasing height, as seen in Fig. 14. Thus, the DDES model has a greater tendency to operate in its RANS mode on the lower part of the tail, and in its LES-mode on the upper tail. The apparent subtle superiority of the DDES result at the VC station is probably not a significant indicator of accuracy, given the discrepancies in $C p$ elsewhere. At the VD station, neither RANS or DDES simulations match the suction spike on the leading edge of the rudder, nor downstream to roughly the middle of the rudder, at $x \sim 170$. The grid is designed to capture the sweep jets, and it may not be as good as needed to resolve the native jets-off turbulence in a separating flow over the rudder hinge and sweep-jet housing.

\section{VI.D. Simulated Sweep-Jet Dynamic Structure}

The preceding results demonstrate that the present simulations can accurately replicate the averaged behavior of the sweep jets, at these conditions. This does not imply that the jets' dynamics are captured well. Nor do we have experimental measurements for this operating condition that will permit an evaluation of the simulated sweep-jet dynamics. This section, therefore, examines the sweep jet structure, with the goal of providing insights without definitive conclusions into dynamical simulation accuracy and the turbulence physics of the flow.

To enhance the ability to visualize the flow, a simulation was run with two gas "species," both of which are air. The second species is injected through the sweep-jet nozzles, and is then used to track the trajectory of the jet fluid. This simulation is at the same condition as above: $\beta=0, \delta_{r}=30^{\circ}$ with MidPSID sweep jets, and run with DDES modeling; the two cases should by aerodynamically identical. Three quantities are used to generate 3D isosurfaces, in an effort to visualize the structure: jet species ( $4 \%$ concentration), local streamwise vorticity $\left(\omega_{i} u_{i} /|u|=0.004\right)$, and stagnation pressure coefficient $C p_{0}=0.7$, shown previously in Fig. 13. (In the freestream, $C p_{0}=1.11$; it is uncorrected for the acceleration due to tunnel-wall boundary layer growth.)

The simplest conceptualization of the jets' dynamical structure is a sinuous trail of jet fluid, imposed on the mean streamwise vortices created by the average $-z$ component of each jet's thrust, which itself exists in the mean 3D flow and the adverse pressure gradient on the rudder. With a frequency of $220 \mathrm{~Hz}$, and a boundary-layer edge Mach number averaged over the length of the rudder of $M \sim 0.2$, the flow should produce a sinuous jet wavelength of roughly $\lambda \sim 12^{\prime \prime}$. If each period of the wavelength yields some form of vortex structure, there should be roughly 7 such structures in the streamwise distance from sweep jet nozzle to the end of the rudder, in the $\sim 80^{\prime \prime}$ rudder chord near the root of the rudder.

Figure 21 gives species and streamwise vorticity isosurfaces. Species and streamwise vorticity isosurfaces tend to show that structures form in the near-field, within 1 jet wavelength $\lambda$ of the nozzle, which then convect downstream while preserving essential features of that structure. The jet species tends to follow a divided path; some of it flows essentially along the mean streamlines near the rudder surface (as inferred from Fig. 17), while the outer jet tends to form into "clumps" of jet fluid that slowly flow root-ward relative to the near-wall jet fluid. Near the root, 7 such clumps along each jet trajectory are visible, while $3 \sim 4$ clumps are seen closer to the tip. The vorticity isosurface show clumps with more complicated structure than seen in the jet species, and in some regions the clumps tend to merge into short bands that span a few vertically adjacent jets. An alternate description of that phenomenon is that short-range phase-coherent arrays appear in the structure, as if there is a "preferred" arrangement of the structures in adjacent jets. However, the initial sweep jets have random phase, so the lattice is not coherent over large areas. Figure 22 shows the streamwise vorticity pattern near the root, at four times over a jet oscillation period. The most dominant structure to the lead author's eyes is an elongated right-handed spiral centered on the streamwise direction. This spiral has outer-layer structures of streamwise vorticity, with a WNW-ESE orientation, and an inner-layer structure with NE-SW orientation. This is a subjective observation.

The flow's ability to resist separation results from the exchange of momentum between the higher-speed outer flow and the lower-speed near-wall flow, and that exchange is affected by the sweep-jets' vortical structure. An isosurface of stagnation pressure is a good means of discriminating between these high- and low-speed regions, especially in the presence of the mean streamwise pressure gradient on the rudder. Figure 23 shows 
isosurfaces of the stagnation pressure coefficient $C p_{0}=0.7$ and streamwise vorticity. The images show the lowest $\sim 10$ sweep jets over the full chord of the rudder; the root of the rudder is visible in the image, for reference. The viewpoint is downstream of the rudder, to help visualize the height of the layer of low $C p_{0}$
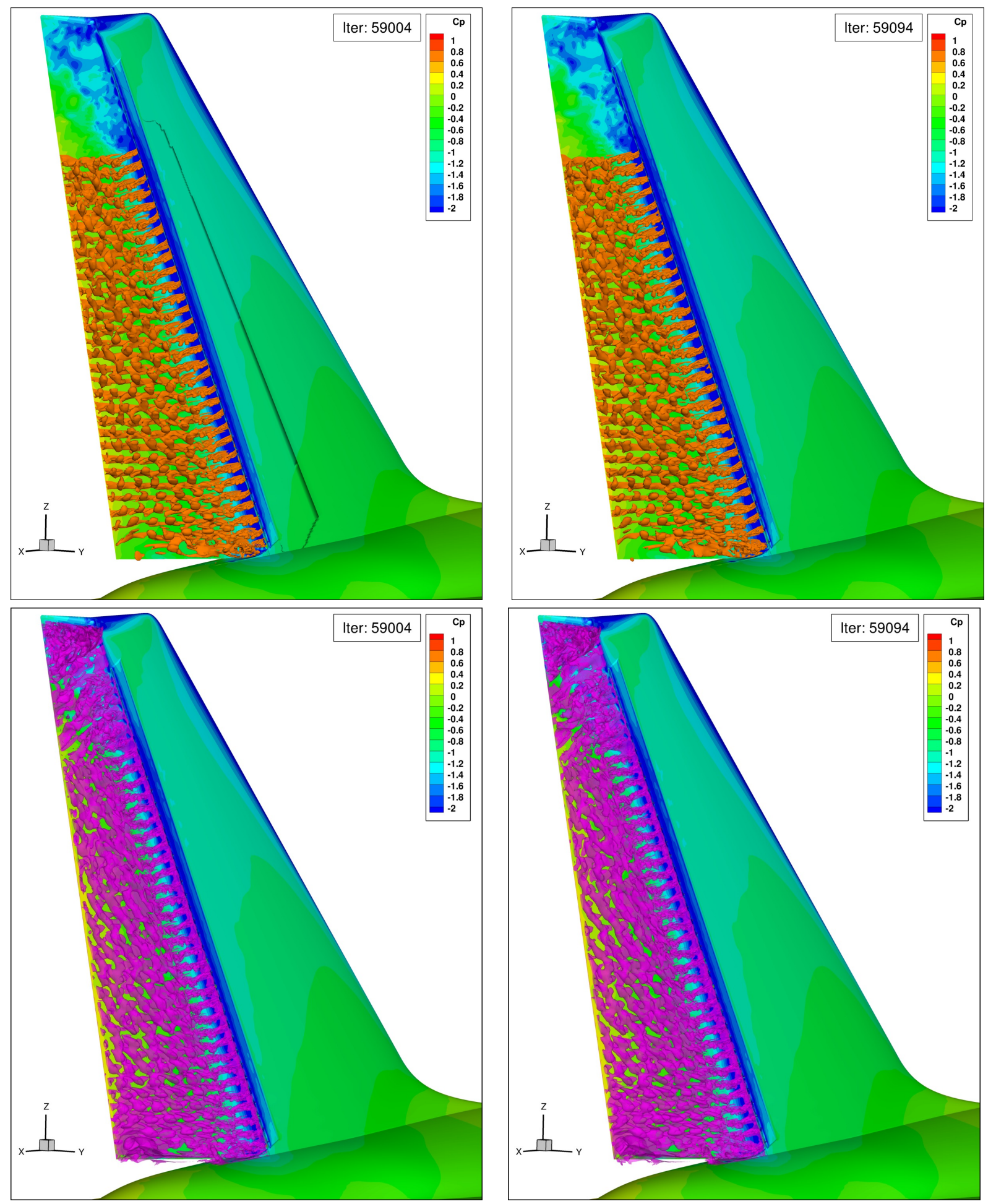

Figure 21: Isosurface of jet "species" concentration (orange, upper images) and local streamwise vorticity (violet, lower images) at two times separated by half a sweep-jet period, $2.3 \mathrm{~ms}$. Contours of $C p$ are given on tail and rudder surface. DDES simulation at $\beta=0$, rudder deflection $\delta_{r}=30^{\circ}$ and with MidPSID sweep jets. 

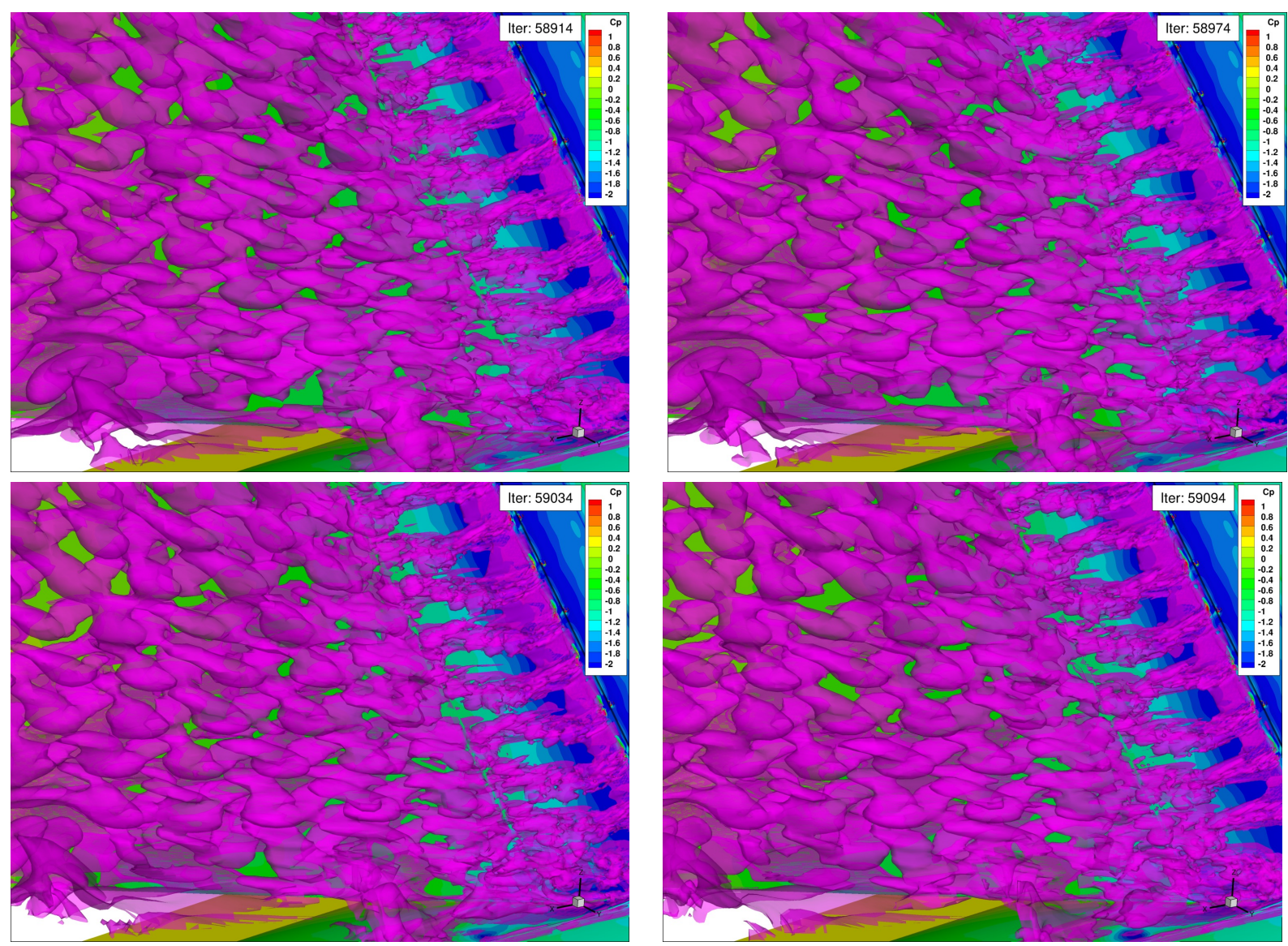

Figure 22: Isosurface local streamwise vorticity, near the rudder root, at four times over one sweep-jet period. Contours of $C p$ are given on tail surface.

on the rudder.

Several features in these images are noted. Near the leading edge of the rudder, where the simulation occurs on the relatively fine near-field and intermediate-field grids, there are small loops or arches in the $C p_{0}$ isosurface; these are vortices on the sweep jets. The vortex cores have lower $C p_{0}$, and the selected level of the isosurface highlights both these loops and the near-wall layer further aft on the rudder. The loops are best seen in the left images, of $C p_{0}$ only, while the right images show that the loops commonly encompass regions of streamwise vorticity. This structure is consistent with turbulent vortices forming on individual jets, and therefore, plausible. The fine-scale turbulence on the finer grids near the nozzles is lost when the flow passes onto the main rudder grid (the lower $\sim 75 \%$ of the visible rudder). On this relatively coarser grid, the streamwise vorticity forms clumps, which tend to align in locally phase-coherent arrays, as in Fig. 21. The $C p_{0}=0.7$ isosurface over the aft rudder displays a structure of streamwise ridges and troughs, which result from the mean streamwise vorticity of the jets, whose mean action is akin to powered vortex generators. There are also roughly periodic streamwise variations on these ridges, which are associated with the dynamic structure in the streamwise vorticity. This streamwise variation in the $C p_{0}=0.7$ height provides a means for the the dynamical structure of the outer flow to transfer energy to the inner flow, which would contribute to suppressing boundary layer separation. Measurements to demonstrate energy transfer from the outer flow to the inner flow, via the observed structure, might involve the phase velocity of the vorticity clumps and the local boundary layer edge velocity, or the correlation between these structures and the fluctuating velocity-pressure gradient work term. Neither measurements have been attempted here.

The implications of these images of the dynamical structure are uncertain, at present. There is clearly a significant loss in the resolved turbulence between the finer near-nozzle grids and the coarser main rudder grid. This loss certainly degrades the accuracy of the simulation, but we cannot know if the essential features of turbulence that suppress boundary layer separation are well represented in the simulation. Because the 

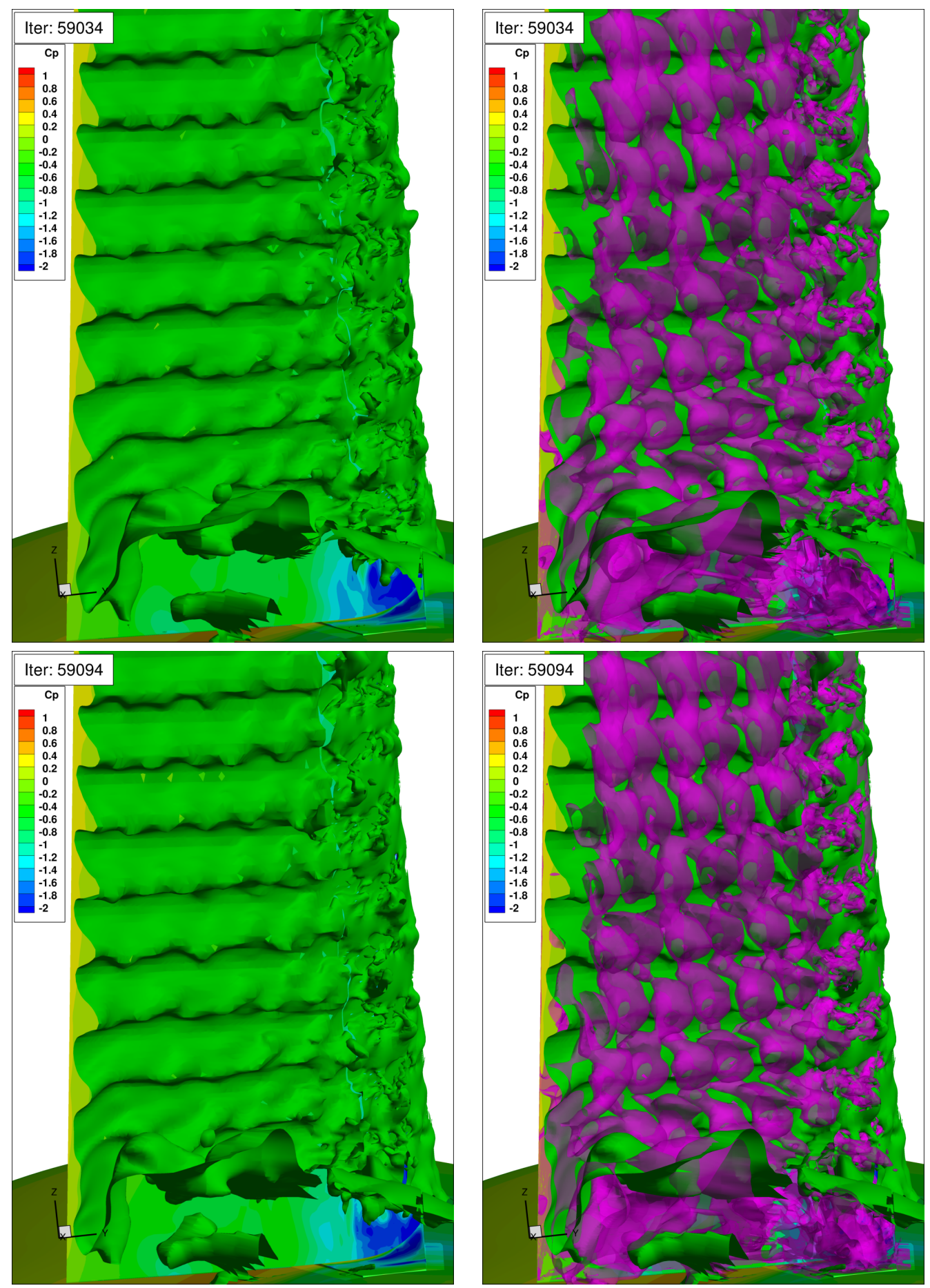

Figure 23: Isosurface of $C p_{0}=0.99$ (green) and local streamwise vorticity (violet). Left frames show just $C p_{0}$ to illustrate the structure of lower-speed flow, and right frames show $C p_{0}$ overlaid by streamwise vorticity.

mean surface pressure distribution matches the experiment, we can only reliably state that the simulation is not obviously inaccurate in a gross sense. Ironically, this loss of small scale turbulence probably helps to simplify the pictures of turbulent structures, as those structures are hard to view when there is a wide range of turbulent scales. In retrospect, the relatively fine intermediate-field grid should be extended to the trailing edge of the rudder. The CPU cost increase will be $O(10 \%)$ of the total cost, and a finer grid would significantly reduce the perceived uncertainty in the results. 


\section{Summary \& Conclusions}

The goal of the present work is to demonstrate the ability of CFD to simulate sweep-jet active flow control devices, which are used to suppress boundary layer separation and improve aerodynamic performance. The present application is a Boeing 757 vertical tail, modified to include an array of 37 sweep jets just upstream of the rudder hinge-line, but practical and effective flow control may be useful in myriad applications. This effort is one element of NASA's Environmentally Responsible Aviation (ERA)project, whose goal is to explore and develop technologies that reduce the adverse environmental effects of aviation.

The focus of the simulation work is a Boeing 757 tail, augmented with sweep-jet flow control that delays the onset of stall to higher rudder deflection angles, relative to a conventional tail. The simulation is developed in steps: first simulations were performed on a single fluidic oscillator and its sweep jet, then the sweep-jet boundary conditions were derived from the single jet simulation, and finally the full tail with many sweep jets was simulated using these boundary conditions. This strategy reduces by an order of magnitude the computational cost, relative to simultaneously simulating 31 fluidic oscillators and the full exterior flow field.

Simulations were performed with Overflow, using fifth-order spatial discretization for convective terms, which is critical to computational efficiency and simulation accuracy. Grid and time-step refinement was performed, based on human judgment of preceived concerns observed in simulation results. The turbulence models considered included the SST-RANS, SST-DES and SST-DDES models. The SST-RANS turbulence model option in Overflow is distinctly superior to SST-DES modeling in predicting the oscillator interior flow. SST-DDES is preferred for the external flow, although we have limited ability to discriminate between it and SST-RANS.

The accuracy of the mean-flow results produced by the simulations appears to be very good, but these are complex flows and the ability to predict the full range of flow control effectiveness is unknown. The close collaboration between experimental work at the Fluid Mechanics Laboratory and the simulation effort was vital to the demonstration of the simulation technology. For the oscillator interior flow, the measured and simulated dominant oscillator frequencies agree very well, but the frequency resolution in the simulation is only $\sim 7 \mathrm{~Hz}$, due to the limited duration of that simulation. The peak-to-peak magnitude of the pressure signal in the sweep-jet throat, simulated with the SST-RANS modeling is about $75 \%$ of the value measured by a Kulite sensor. In the external sweep jet, there are roughly $20 \% \sim 30 \%$ differences in the mean velocities and lateral-spreading turbulent shear stress, as measured by PIV (particle imaging velocimetry) and simulated with the SST-DDES model.

Accuracy for the mean-flow characteristics on the full Boeing 757 tail simulation is very good, at the conditions simulated. The integrated loads agree well with measurements at the high jet blowing rates which achieved the Project goal of good performance augmentation. The surface pressure distributions were also very good, especially in the critical region of the suction surface of the rudder where the sweep jets were powered. The largest discrepancy between simulation and experiment in the measured surface pressures occurred in the separated flow on the upper tail where the sweep jets were unpowered. It is important to recognize that where the rudder boundary layer remains attached due to the sweep jets, all simulations that achieve attached flow could give similarly good results. For example, simulations with the (slip-wall) Euler-equations fall into this category. A more rigorous test of accuracy of these types of simulations will occur under a range of flow conditions that display varying levels of flow separation on the rudder.

The ability of the simulations to accurately portray the dynamical vortex structure induced by the sweep jets is a key step to understanding and achieving good flow-control performance. We cannot here validate the accuracy of the predicted vortex structure, and the present observations are just an initial step toward a good assessment of this topic. These structures are observed to be large enough that the eddies from neighboring jets interact strongly. Short-range order among the eddies was observed, in which, presumably, the neighboring eddies coexist without a rapid cascade of turbulence energy to heat. The ability to generate persistent structures that are also effective at mixing near-wall and outer-layer fluid is expected to be a critical feature of successful flow control.

Acknowledgments This work was made possible by the NASA Environmentally Responsible Aviation Project; Principle Investigator: John Lin; Project Management: Fay Collier, Tony Washburn, Michael 
Alexander, and Robert Fong; and Ames Research Center's S. Pete Worden. Significant work on the tail's CAD description was done by Veronica Hawke. The simulations were run on NASA's Pleiades computer system.

\title{
References
}

\author{
${ }^{1}$ http://www.aeronautics.nasa.gov/iasp/era/index.htm "NASA ERA Project" \\ ${ }^{2}$ http://www.nasa.gov/aero/nasa-tests-green-aviation-technology-on-boeing-ecodemonstrator.html "NASA Tests Green Avia- \\ tion Technology on Boeing ecoDemonstrator" \\ ${ }^{3}$ Andino, M.Y., Lin, John C., Washburn, A.E., Whalen, E.A., Graff, E., Wygnanski, I.J., "Flow Separation Control on a \\ Full-Scale Vertical Tail Model using Sweeping Jet Actuators" AIAA 2015-0785 \\ ${ }^{4}$ Bres, G., Fares, E., Williams, D., and Colonius, T., "Numerical Simulations of the Transient Flow Response of a 3D, Low- \\ Aspect-Ratio Wing to Pulsed Actuation," AIAA-2011-3440. \\ ${ }^{5}$ DeSalvo, M., Whalen, E., and Glezer, A., "High-Lift Enhancement using Fluidic Actuation," AIAA-2010-0863.
}

${ }^{6}$ Gatski, T. and Rumsey, C., "CFD Validation of Synthetic Jets and Turbulent Separation Control," http://cfdval2004.larc.nasa.gov

${ }^{7}$ Gokoglu, S., Kuczmarski, M., Culley, D., and Raghu, S., "Numerical Studies of a Fluidic Diverter for Flow Control," AIAA2009-4012.

${ }^{8}$ Gokoglu, S., Kuczmarski, M., Culley, D., and Raghu, S., "Numerical Studies of a Supersonic Fluidic Diverter Actuator for Flow Control," AIAA-2010-4415.

${ }^{9}$ Gokoglu, S., Kuczmarski, M., Culley, D., and Raghu, S., "Numerical Studies of an Array of Fluidic Diverter Actuators for Flow Control," AIAA Paper 2011-3100.

${ }^{10}$ Kushner, L.K., Heineck, J.T., Storms, B.L., and Childs, R.E., "Visualization of a Sweeping Jet by Laser Speckle Retroreflective Background Oriented Schlieren," AIAA-2015-1697

${ }^{11}$ Menter, Florian R. "Improved two-equation k-omega turbulence models for aerodynamic flows." NASA STI/Recon Technical Report N 93 (1992): 22809.

${ }^{12}$ Menter, F. R., Kuntz, M., and Langtry, R., "Ten Years of Industrial Experience with the SST Turbulence Model," Turbulence, Heat and Mass Transfer 4, ed: K. Hanjalic, Y. Nagano, and M. Tummers, Begell House, Inc., 2003, pp. 625 - 632.

${ }^{13}$ Mort, Kenneth, W., "Boundary Layers and Wall Pressures in 40-by-80-Ft. Wind Tunnel with Deep Cavity Acoustic Lining" Prepared for NASA/Ames Research Center, BPA/54869D(MXD), Call No. 54, Aug. 28, 2000.

${ }^{14}$ Seele, R. Graff, E., Gharib, M., Taubert, L., Lin, John, and Wygnanski, I., "Improving Rudder Effectiveness with Sweeping Jet Actuators," AIAA 2012-3244

${ }^{15}$ Shmilovich, A., Yadlin, Y., and Whalen, E., "Computational Evaluation of Flow Control for Enhanced Control Authority of a Vertical Tail" AIAA-2015-3311

${ }^{16}$ Suhs, N. E. Rogers, S. E., and Dietz, W. E. "PEGASUS 5: An Automated Pre-processor for Overset-Grid CFD," AIAA Paper 2002-3186, AIAA Fluid Dynamics Conference, June 2002, St. Louis, MO.

${ }^{17}$ Tramel, R.W., Nichols R.H., and Buning P.G., "Addition of Improved Shock-Capturing Schemes to OVERFLOW 2.1" AIAA-2009-3988.

${ }^{18}$ Vatsa, V.N., Koklu, M., Wygnanski, I., Fares E., "Numerical Simulation of Fluidic Actuators for Flow Control Applications," AIAA-2012-3239.

${ }^{19}$ Vatsa, V.N., Casalino, D. Lin, J.C., Appelbaum, J., "Numerical Simulation of a High-Lift Configuration with Embedded Fluidic Actuators" AIAA-2014-2142.

${ }^{20}$ Whalen, E.A. and Goldhammer, M.I. "Active Flow Control on a Vertical Stabilizer and Rudder," United States Patent, 2012/0091266 A1, 2012.

${ }^{21}$ Whalen, E.A., Lacy, D., Lin, John C., Andino, M.Y., Washburn, A.E., Graff, E., Wygnanski, I.J., "Performance Enhancement of a Full-Scale Vertical Tail Model Equipped with Active Flow Control" AIAA-2015-0784

${ }^{22}$ Whalen, E.A., Khodadoust, A., "Final Report, Flight Services and Aircraft Access, NASA NAS1-NNL10AA05B TASK NNL14AA57T" The Boeing Company, August 31, 2015

${ }^{23}$ Woszidlo, R. and Wygnanski, I., "Parameters Governing Separation Control with Sweeping Jet Actuators," AIAA-2011-3172. 